\title{
Elastic wave scattering by periodic structures of spherical objects: Theory and experiment
}

\author{
Zhengyou Liu, C. T. Chan, and Ping Sheng \\ Department of Physics, The Hong Kong University of Science and Technology, Clear Water Bay, Kowloon, Hong Kong, China \\ A. L. Goertzen* and J. H. Page \\ Department of Physics, University of Manitoba, Winnipeg, Manitoba, R3T 2N2, Canada
}

(Received 22 November 1999; revised manuscript received 17 April 2000)

\begin{abstract}
We extend the multiple-scattering theory for elastic waves by taking into account the full vector character. The formalism for both the band structure calculation and the reflection and transmission calculations for finite slabs is presented. The latter is based on a double-layer scheme which obtains the reflection and transmission matrix elements for the multilayer slab from those of a single layer. As a demonstration of applications of the formalism, we calculate the band structures of elastic waves propagating in a three-dimensional periodic arrangement of spherical particles and voids, as well as the transmission coefficients through finite slabs. In contrast with the plane-wave method, the multiple-scattering approach exhibits advantages in handling specialized geometries (spherical geometry in the present case). We also present a comparison between theory and ultrasound experiment for a hexagonal-close-packed array of steel balls immersed in water. Excellent agreement is obtained.
\end{abstract}

\section{INTRODUCTION}

In recent years, there has been growing interest in classical wave propagation in periodic composite media. ${ }^{1-3}$ The study of photonic crystals has led the way, ${ }^{1-3}$ with the theoretical prediction ${ }^{1-3}$ and experimental realization of photonic band gaps. ${ }^{1}$ Recently the focus has been extended to the study of acoustic and elastic waves in periodic media. ${ }^{4-8}$ Currently, all elastic wave band structure calculations are based on the plane-wave (PW) approach. ${ }^{4-8}$ The PW method exhibits flexibility in handling different types of periodic structure, but has convergence problems when dealing with systems of either very high or very low filling ratios. The PW method is also less effective when dealing with disordered systems. Multiple-scattering theory (MST), ${ }^{9}$ through its success in electronic structure calculations for both ordered and disordered systems, shows great promise in complementing the PW approach for the study of elastic wave scattering and propagation in both ordered and disordered media. MST, usually known as the KKR (Korringa, Kohn, and Rostoker) approach, was developed mainly for the calculation of electronic band structures, ${ }^{9}$ although it originated from the study of classical waves (including acoustic waves). ${ }^{9}$ MST, in the spirit of the KKR approach, has been developed for the electromagnetic wave, and was successfully applied to the band structure calculation of photonic crystals. ${ }^{3}$ At the same time, a layer MST theory ${ }^{10}$ of electromagnetic waves was also successfully implemented, ${ }^{11}$ thus enabling rigorous calculation of the transmission and reflection coefficients for a slab of periodically arranged scatterers, and providing a direct way to compare theory with experiment. In this paper, we present a rigorous multiplescattering formalism for calculating the band structure of elastic systems, and further extend the layer MST theory to the elastic wave case.

In what follows, the MST equations for the elastic waves are presented in Sec. II, and the low-energy electron diffrac- tion theory for elastic waves is presented in Secs. III and IV. In Sec. V, we use two sets of examples to demonstrate the application of our formalism. In Sec. VI, we give a comparison between theory and ultrasound experiment on a system of hexagonal-close-packed steel balls immersed in water. Excellent agreement is obtained. Derivation of some identities is given in the Appendixes.

\section{MULTIPLE-SCATTERING THEORY FOR ELASTIC WAVES}

Multiple scattering of elastic waves by particles has been extensively studied during the last 20 years, ${ }^{12-14}$ and the scattering of elastic waves by a periodic array of scatterers has also been studied by some authors. ${ }^{15,16}$ In this section, we present MST for elastic waves in its modern form, and formulates the MST equations so that they are convenient for use in numerical calculations.

In a homogeneous medium, the elastic wave equation may be written as

$$
(\lambda+2 \mu) \boldsymbol{\nabla}(\boldsymbol{\nabla} \cdot \mathbf{u})-\mu \boldsymbol{\nabla} \times \boldsymbol{\nabla} \times \mathbf{u}+\rho \omega^{2} \mathbf{u}=\mathbf{0},
$$

where $\rho$ is the density and $\lambda, \mu$ are the Lamé constants of the medium. In spherical coordinates, the general solution can be expressed as

$$
\mathbf{u}(\mathbf{r})=\sum_{l m \sigma}\left[a_{l m \sigma} \mathbf{J}_{l m \sigma}(\mathbf{r})+b_{l m \sigma} \mathbf{H}_{l m \sigma}(\mathbf{r})\right],
$$

where $\mathbf{J}_{l m \sigma}(\mathbf{r}), \mathbf{H}_{l m \sigma}(\mathbf{r})$ are defined as

$$
\begin{gathered}
\mathbf{J}_{l m 1}(\mathbf{r})=\frac{1}{\alpha} \nabla\left[j_{l}(\alpha r) Y_{l m}(\hat{\mathbf{r}})\right], \\
\mathbf{J}_{l m 2}(\mathbf{r})=\frac{1}{\sqrt{l(l+1)}} \nabla \times\left[\mathbf{r} j_{l}(\beta r) Y_{l m}(\hat{\mathbf{r}})\right],
\end{gathered}
$$




$$
\mathbf{J}_{l m 3}(\mathbf{r})=\frac{1}{\sqrt{l(l+1)} \beta} \nabla \times \nabla \times\left[\mathbf{r} j_{l}(\beta r) Y_{l m}(\hat{\mathbf{r}})\right],
$$

and

$$
\begin{gathered}
\mathbf{H}_{l m 1}(\mathbf{r})=\frac{1}{\alpha} \boldsymbol{\nabla}\left[h_{l}(\alpha r) Y_{l m}(\hat{\mathbf{r}})\right], \\
\mathbf{H}_{l m 2}(\mathbf{r})=\frac{1}{\sqrt{l(l+1)}} \boldsymbol{\nabla} \times\left[\mathbf{r} h_{l}(\beta r) Y_{l m}(\hat{\mathbf{r}})\right], \\
\mathbf{H}_{l m 3}(\mathbf{r})=\frac{1}{\sqrt{l(l+1)} \beta} \nabla \times \nabla \times\left[\mathbf{r} h_{l}(\beta r) Y_{l m}(\hat{\mathbf{r}})\right],
\end{gathered}
$$

where $\alpha=\omega \sqrt{\rho / \lambda+2 \mu}, \beta=\omega \sqrt{\rho / \mu}, j_{l}(x)$ is the spherical Bessel function, and $h_{l}(x)$ is the spherical Hankel function of the first kind. In Eq. (2), the index $\sigma$, ranging from 1 to 3, stands for three kinds of modes $-\sigma=1$ is for the longitudinal mode, and $\sigma=2,3$ represent the two transverse modes. Except for the normalization the $\mathbf{J}$ and $\mathbf{H}$ functions are the same as the $\mathbf{L}, \mathbf{M}$, and $\mathbf{N}$ functions employed by Morse and Feshbach ${ }^{17}$ and the $\psi$ functions by Waterman. ${ }^{12}$ When the coefficients $b_{l m \sigma}$ are zero, $\mathbf{u}(\mathbf{r})$ represents the incoming wave, and $a_{l m \sigma}=0$ implies that $\mathbf{u}(\mathbf{r})$ consists of only the outgoing wave. In a composite medium, the displacement in each homogeneous region obeys Eq. (1), and can thus be expressed in the form of Eq. (2). By regarding the composite medium as composed of a host matrix and embedded scatterers, the incident wave for scatterer $i$ may be expressed as

$$
\mathbf{u}_{i}^{i n}\left(\mathbf{r}_{i}\right)=\sum_{l m \sigma} a_{l m \sigma}^{i} \mathbf{J}_{l m \sigma}^{i}\left(\mathbf{r}_{i}\right)
$$

where $\mathbf{r}_{i}$ is measured from the center of scatterer $i$. The wave scattered by scatterer $i$ may be expressed as

$$
\mathbf{u}_{i}^{s c}\left(\mathbf{r}_{i}\right)=\sum_{l m \sigma} b_{l m \sigma}^{i} \mathbf{H}_{l m \sigma}^{i}\left(\mathbf{r}_{i}\right)
$$

According to MST, the wave incident on a given scatterer consists of two parts. One is the externally incident wave $\mathbf{u}_{i}^{i n(0)}\left(\mathbf{r}_{i}\right)$, which may be expanded as

$$
\mathbf{u}_{i}^{i n(0)}\left(\mathbf{r}_{i}\right)=\sum_{l m \sigma} a_{l m \sigma}^{i(0)} \mathbf{J}_{l m \sigma}^{i}\left(\mathbf{r}_{i}\right) .
$$

The second part is the sum of all the scattered waves except that from scatterer $i$, given by

$$
\mathbf{u}_{i}^{i n}\left(\mathbf{r}_{i}\right)-\mathbf{u}_{i}^{i n(0)}\left(\mathbf{r}_{i}\right)=\sum_{j \neq i} \sum_{l^{\prime \prime} m^{\prime \prime} \sigma^{\prime \prime}} b_{l^{\prime \prime} m^{\prime \prime} \sigma^{\prime \prime}}^{j} \mathbf{H}_{l^{\prime \prime} m^{\prime \prime} \sigma^{\prime \prime}}^{j}\left(\mathbf{r}_{j}\right)
$$

where $\mathbf{r}_{i}$ and $\mathbf{r}_{j}$ refer to the position of the same spatial point measured from scatterers $i$ and $j$, respectively. With $\mathbf{R}_{i(j)}$ denoting the position of scatterer $i(j)$, we have $\mathbf{r}_{j}=\mathbf{r}_{i}+\mathbf{R}_{i}$ $-\mathbf{R}_{j}$. It may be proved that

$$
\mathbf{H}_{l^{\prime \prime} m^{\prime \prime} \sigma^{\prime \prime}}^{j}\left(\mathbf{r}_{i}+\mathbf{R}_{i}-\mathbf{R}_{j}\right)=\sum_{l m \sigma} G_{l^{\prime \prime} m^{\prime \prime} \sigma^{\prime \prime} l m \sigma}\left(\mathbf{R}_{i}-\mathbf{R}_{j}\right) \mathbf{J}_{l m \sigma}^{i}\left(\mathbf{r}_{i}\right)
$$

(see Appendix A for details). Where $G$ is the so-called vector structure constant, which is essentially the translation matrix given by Bostrom, ${ }^{13}$ and is given by

$$
G_{l m \sigma l^{\prime} m^{\prime} \sigma^{\prime}}(\mathbf{R})= \begin{cases}X_{l m l^{\prime} m^{\prime}}^{\alpha}(\mathbf{R}), & \sigma=\sigma^{\prime}=1 \\ \sum_{\mu} c(l 1 l m-\mu \mu) X_{l m-\mu l^{\prime} m^{\prime}-\mu^{\prime}}^{\beta}(\mathbf{R}) c\left(l^{\prime} 1 l^{\prime} m^{\prime}-\mu \mu\right), & \sigma=\sigma^{\prime}=2,3 \\ -i\left(\frac{2 l^{\prime}+1}{l^{\prime}+1}\right)^{1 / 2} \sum_{\mu} c(l 1 l m-\mu \mu) X_{l m-\mu l^{\prime}-1 m^{\prime}-\mu^{\prime}}^{\beta}(\mathbf{R}) c\left(l^{\prime}-11 l^{\prime} m^{\prime}-\mu \mu\right), & \sigma \neq \sigma^{\prime} ; \sigma, \sigma^{\prime} \neq 1 .\end{cases}
$$

$X_{l m l^{\prime} m^{\prime}}^{\kappa}(\mathbf{R})$ is the so-called structure constant for scalar waves, defined as

$$
\begin{aligned}
X_{l m l^{\prime} m^{\prime}}^{\kappa}(\mathbf{R}) & \\
= & 4 \pi \sum_{l^{\prime \prime}} i^{l^{\prime}+l^{\prime \prime}-l} C_{l^{\prime} m^{\prime} l^{\prime \prime} m-m^{\prime}}^{l m} h_{l^{\prime \prime}}(\kappa R) Y_{l^{\prime \prime} m-m^{\prime}}(\hat{\mathbf{R}}) .
\end{aligned}
$$

Here $C_{l^{\prime} m^{\prime} l^{\prime \prime} m^{\prime \prime}}^{l m}$ is an integral,

$$
C_{l^{\prime} m^{\prime} l^{\prime \prime} m^{\prime \prime}}^{l m}=\iint_{S} Y_{l m}(\Omega) Y_{l^{\prime} m^{\prime}}^{*}(\Omega) Y_{l^{\prime \prime} m^{\prime \prime}}^{*}(\Omega) d \Omega .
$$

By defining $G_{l^{\prime \prime} m^{\prime \prime} \sigma^{\prime \prime l m} \sigma}^{i j}=G_{l^{\prime \prime} m^{\prime \prime} \sigma^{\prime \prime} l m \sigma}\left(\mathbf{R}_{i}-\mathbf{R}_{j}\right), \mathbf{H}_{l^{\prime \prime} m^{\prime \prime} \sigma^{\prime \prime}}^{j}\left(\mathbf{r}_{j}\right)$ may be expressed as

$$
\mathbf{H}_{l^{\prime \prime} m^{\prime \prime} \sigma^{\prime \prime}}^{j}\left(\mathbf{r}_{j}\right)=\sum_{l m \sigma} G_{l^{\prime \prime} m^{\prime \prime} \sigma^{\prime \prime} l m \sigma}^{i j} \mathbf{J}_{l m \sigma}^{i}\left(\mathbf{r}_{i}\right) .
$$

For a given scatterer, the scattered displacement field is completely determined from the incident field through the scattering matrix. There is a relation between the expansion coefficients $A=\left\{b_{l m \sigma}^{j}\right\}$ and $B=\left\{a_{l m \sigma}^{j}\right\}$ :

$$
B=T A,
$$


where the scattering matrix $T=\left\{t_{l m \sigma l^{\prime} m^{\prime} \sigma^{\prime}}\right\}$ can be obtained from the elastic Mie scattering solution of a scatterer. ${ }^{12}$ Substituting Eqs. (5), (7), (13), and (14) into Eq. (8), we arrive at

$$
\begin{aligned}
& \sum_{j l^{\prime} m^{\prime} \sigma^{\prime}}\left(\delta_{i j} \delta_{l l^{\prime}} \delta_{m m^{\prime}} \delta_{\sigma \sigma^{\prime}}\right. \\
& \left.\quad-\sum_{l^{\prime \prime} m^{\prime \prime} \sigma^{\prime \prime}} t_{l^{\prime \prime} m^{\prime \prime} \sigma^{\prime \prime} l^{\prime} m^{\prime} \sigma^{\prime}}^{j} G_{l^{\prime \prime} m^{\prime \prime} \sigma^{\prime \prime} l m \sigma}^{i j}\right) a_{l^{\prime} m^{\prime} \sigma^{\prime}}^{j}=a_{l m \sigma}^{i(0)} .
\end{aligned}
$$

This is the final equation for a multiple-scattering system. It has the general form of the scalar KKR theory. For a finite and/or disordered system, we must solve this equation in order to investigate the system response to external perturbations. The normal modes of the system may be obtained by solving the following secular equation, in the absence of an external incident wave:

$$
\operatorname{det}\left|\delta_{i j} \delta_{l l^{\prime}} \delta_{m m^{\prime}} \delta_{\sigma \sigma^{\prime}}-\sum_{l^{\prime \prime} m^{\prime \prime} \sigma^{\prime \prime}} t_{l^{\prime \prime} m^{\prime \prime} \sigma^{\prime \prime} l^{\prime} m^{\prime} \sigma^{\prime}}^{j} G_{l^{\prime \prime} m^{\prime \prime} \sigma^{\prime \prime} l m \sigma}^{i j}\right|=0 .
$$

For a periodic system, Eq. (16) may be transformed to

$$
\begin{aligned}
\operatorname{det} \mid & \delta_{s s^{\prime}} \delta_{l l^{\prime}} \delta_{m m^{\prime}} \delta_{\sigma \sigma^{\prime}}-\sum_{l^{\prime \prime} m^{\prime \prime} \sigma^{\prime \prime}} t_{l^{\prime \prime} m^{\prime \prime} \sigma^{\prime \prime} l^{\prime} m^{\prime} \sigma^{\prime}}^{s^{\prime}} G_{l^{\prime \prime} m^{\prime \prime} \sigma^{\prime \prime} l m \sigma}^{s s^{\prime}}(\mathbf{k}) \mid \\
& =0
\end{aligned}
$$

where $s$ and $s^{\prime}$ label the scatterers in the unit cell with position vectors $\mathbf{0}_{s}$ and $\mathbf{o}_{s}^{\prime}$, and $G_{l^{\prime \prime} m^{\prime \prime} \sigma^{\prime \prime} l m \sigma}^{s \sigma^{\prime}}(\mathbf{k})$ is defined as

$$
G_{l^{\prime \prime} m^{\prime \prime} \sigma^{\prime \prime} l m \sigma}^{s s^{\prime}}(\mathbf{k})=\sum_{\mathbf{R}} G_{l^{\prime \prime} m^{\prime \prime} \sigma^{\prime \prime} l m \sigma}\left(\mathbf{o}_{s}-\mathbf{o}_{s^{\prime}}-\mathbf{R}\right) \exp (i \mathbf{k} \cdot \mathbf{R}),
$$

where the sum $\Sigma_{\mathbf{R}}$ is over all lattice sites. The solution of Eq. (17) gives the band structure of an elastic periodic system.

\section{ELASTIC WAVE SCATTERING BY A PLANAR LAYER OF SCATTERERS}

The study of elastic wave scattering by a periodic array of scatterers has a long history. ${ }^{15,16}$ In this section and the next, we formulate MST in a layer-by-layer approach for calculating the transmission and reflection from a finite slab of periodically arranged scatterers. The formulation is an extension of the formalism of Modinos and co-workers ${ }^{11}$ developed for electron and electromagnetic waves, starting with the problem of elastic wave scattering by a planar layer of scatterers. In this case, the scatterers are located on sites $\left\{\mathbf{R}_{n}\right\}$ of a two-dimensional lattice in the $x-y$ plane, i.e.,

$$
\mathbf{R}_{n}=n_{1} \mathbf{a}_{1}+n_{2} \mathbf{a}_{2},
$$

where $\mathbf{a}_{1}$ and $\mathbf{a}_{2}$ are primitive vectors in the $x-y$ plane, and $n_{1}, n_{2}$ are integers. For reference, the $z$ axis is assumed to point to the left of the $x-y$ plane. A plane elastic wave incident on the scatterers may be expressed in general as

$$
\begin{gathered}
\mathbf{u}_{\alpha}^{i n \pm}(\mathbf{r})=\sum_{\mathbf{g}} \mathbf{u}_{\alpha g}^{i n \pm}(\mathbf{r})=\sum_{\mathbf{g}} \mathbf{U}_{\alpha g}^{i n \pm} \exp \left(i \mathbf{k}_{\alpha g}^{ \pm} \cdot \mathbf{r}\right), \\
\mathbf{U}_{\alpha g}^{i n \pm} \times \mathbf{k}_{\alpha g}^{ \pm}=\mathbf{0}, \\
\mathbf{u}_{\beta}^{i n \pm}(\mathbf{r})=\sum_{\mathbf{g}} \mathbf{u}_{\beta g}^{i n \pm}(\mathbf{r})=\sum_{\mathbf{g}} \mathbf{U}_{\beta g}^{i n \pm} \exp \left(i \mathbf{k}_{\beta g}^{ \pm} \cdot \mathbf{r}\right), \\
\mathbf{k}_{\alpha g}^{ \pm}=\left(\mathbf{k}_{\beta g}^{i n \pm}+\mathbf{g}, \pm \sqrt{\alpha^{2}-\left|\mathbf{k}_{\|}+\mathbf{g}\right|^{2}}\right), \\
\mathbf{k}_{\beta g}^{ \pm}=\left(\mathbf{k}_{\|}+\mathbf{g}, \pm \sqrt{\beta^{2}-\left|\mathbf{k}_{\|}+\mathbf{g}\right|^{2}}\right),
\end{gathered}
$$

where $\mathbf{u}_{\alpha}^{i n \pm}(\mathbf{r})$ and $\mathbf{u}_{\beta}^{\text {in } \pm}(\mathbf{r})$ represent the longitudinal and transverse plane elastic waves, respectively, the sign + means incident from the left of the plane (positive $z$ ), and - means incident from the right of the plane (negative $z$ ). Here, $\mathbf{g}$ is one of the two-dimensional (2D) reciprocal lattice vectors in the plane of the scatterers, and $\mathbf{k}_{\|}$is a reduced wavevector in the 2D Brillouin zone of the reciprocal lattice. Thus, the incident plane elastic waves may be expressed as

$$
\mathbf{u}^{i n}(\mathbf{r})=\mathbf{u}_{\alpha}^{i n}(\mathbf{r})+\mathbf{u}_{\beta}^{i n}(\mathbf{r})=\sum_{s \mathbf{g}} \mathbf{u}_{\alpha g}^{i n s}(\mathbf{r})+\sum_{s \mathbf{g}} \mathbf{u}_{\beta g}^{i n s}(\mathbf{r}) .
$$

The incident elastic waves may be expanded in the spherical coordinate basis:

$$
\mathbf{u}^{i n}(\mathbf{r})=\sum_{l m \sigma} a_{l m \sigma} \mathbf{J}_{l m \sigma}(\mathbf{r})
$$

or, more explicitly,

$$
\mathbf{u}_{\alpha}^{i n}(\mathbf{r})=\sum_{l m} a_{l m 1} \mathbf{J}_{l m 1}(\mathbf{r})
$$

$$
\mathbf{u}_{\beta}^{i n}(\mathbf{r})=\sum_{l m} a_{l m 2} \mathbf{J}_{l m 2}(\mathbf{r})+a_{l m 3} \mathbf{J}_{l m 3}(\mathbf{r})
$$

where the coefficients $a_{l m \sigma}$, derived from $\mathbf{U}_{\alpha g}^{i n \pm}$ and $\mathbf{U}_{\beta g}^{i n \pm}$ (see Appendix B for the details), may be expressed as

$$
\begin{aligned}
& a_{l m 1}=\sum_{s \mathbf{g}} \mathbf{U}_{\alpha g}^{i n s} \cdot \mathbf{A}_{l m 1}^{g s}, \\
& a_{l m 2}=\sum_{s \mathbf{g}} \mathbf{U}_{\beta g}^{i n s} \cdot \mathbf{A}_{l m 2}^{g s}, \\
& a_{l m 3}=\sum_{s \mathbf{g}} \mathbf{U}_{\beta g}^{i n s} \cdot \mathbf{A}_{l m 3}^{g s},
\end{aligned}
$$

with $\mathbf{A}_{l m \sigma}^{g \pm}$ defined as

$$
\mathbf{A}_{l m 1}^{g \pm}=\frac{4 \pi i^{l-1}(-1)^{m}}{\alpha} Y_{l-m}\left(\hat{\mathbf{k}}_{\alpha g}^{ \pm}\right) \hat{\mathbf{k}}_{\alpha g}^{ \pm},
$$




$$
\begin{aligned}
\mathbf{A}_{l m 2}^{g \pm}= & \frac{4 \pi i^{l+1}(-1)^{m+1}}{\sqrt{l(l+1)}}\left\{\left[M_{l}^{m} Y_{l-(m+1)}\left(\hat{\mathbf{k}}_{\beta g}^{ \pm}\right)\right.\right. \\
& \left.+N_{l}^{m} Y_{l-(m-1)}\left(\hat{\mathbf{k}}_{\beta g}^{ \pm}\right)\right] \hat{\mathbf{x}}+i\left[M_{l}^{m} Y_{l-(m+1)}\left(\hat{\mathbf{k}}_{\beta g}^{ \pm}\right)\right. \\
& \left.\left.-N_{l}^{m} Y_{l-(m-1)}\left(\hat{\mathbf{k}}_{\beta g}^{ \pm}\right)\right] \hat{\mathbf{y}}-m Y_{l-m}\left(\hat{\mathbf{k}}_{\beta g}^{ \pm}\right) \hat{\mathbf{z}}\right\}, \\
\mathbf{A}_{l m 3}^{g \pm}= & \frac{4 \pi i^{l}(-1)^{m+1}}{\sqrt{l(l+1)} \beta} \mathbf{k}_{\beta g}^{ \pm} \times\left\{\left[M_{l}^{m} Y_{l-(m+1)}\left(\hat{\mathbf{k}}_{\beta g}^{ \pm}\right)\right.\right. \\
& \left.+N_{l}^{m} Y_{l-(m-1)}\left(\hat{\mathbf{k}}_{\beta g}^{ \pm}\right)\right] \hat{\mathbf{x}}+i\left[M_{l}^{m} Y_{l-(m+1)}\left(\hat{\mathbf{k}}_{\beta g}^{ \pm}\right)\right. \\
& \left.\left.-N_{l}^{m} Y_{l-(m-1)}\left(\hat{\mathbf{k}}_{\beta g}^{ \pm}\right)\right] \hat{\mathbf{y}}-m Y_{l-m}\left(\hat{\mathbf{k}}_{\beta g}^{ \pm}\right) \hat{\mathbf{z}}\right\},
\end{aligned}
$$

and $M_{l}^{m}$ and $N_{l}^{m}$ given by

$$
\begin{aligned}
& M_{l}^{m}=\frac{1}{2} \sqrt{(l-m)(l+m+1)}, \\
& N_{l}^{m}=\frac{1}{2} \sqrt{(l+m)(l-m+1)} .
\end{aligned}
$$

In general, the wave scattered by a scatterer $i$ may be expanded as $\Sigma_{l m \sigma} b_{l m \sigma}^{i} \mathbf{H}_{l m \sigma}^{i}\left(\mathbf{r}_{i}\right)$, which is completely determined by the incident waves plus the scatterer parameters and geometry. The total scattered wave contains contributions from all the scatterers in the plane:

$$
\mathbf{u}^{s c}(\mathbf{r})=\sum_{i l m \sigma} b_{l m \sigma}^{i} \mathbf{H}_{l m \sigma}^{i}\left(\mathbf{r}_{i}\right)
$$

According to the Bloch theorem, we have

$$
\mathbf{u}^{s c}(\mathbf{r})=\sum_{l m \sigma} b_{l m \sigma} \sum_{\mathbf{R}} \exp \left(i \mathbf{k}_{\|} \cdot \mathbf{R}\right) \mathbf{H}_{l m \sigma}(\mathbf{r}-\mathbf{R}),
$$

where $\left\{b_{l m \sigma}\right\}$ are the expansion coefficients (defined above) for the central scatterer, with the superscript omitted. It may be proved (see Appendix $\mathrm{C}$ for the details) that

$$
\begin{gathered}
B=Z A, \\
Z=\left[I-T G^{T r}\left(\mathbf{k}_{\|}\right)\right]^{-1} T,
\end{gathered}
$$

where $A=\left\{a_{l m \sigma}\right\}, B=\left\{b_{l m \sigma}\right\}, T=\left\{t_{l m \sigma l^{\prime} m^{\prime} \sigma^{\prime}}\right\}$ is the scattering matrix for a single scatterer, and $G=\left\{G_{l m \sigma l^{\prime} m^{\prime} \sigma^{\prime}}\left(\mathbf{k}_{\|}\right)\right\}$ with

$$
G_{l m \sigma l^{\prime} m^{\prime} \sigma^{\prime}}\left(\mathbf{k}_{\|}\right)=\sum_{\mathbf{R}} \exp \left(i \mathbf{k}_{\|} \cdot \mathbf{R}\right) G_{l m \sigma l^{\prime} m^{\prime} \sigma^{\prime}}(-\mathbf{R}) .
$$

Here the sum over $\mathbf{R}$ covers the whole two-dimensional lattice, excluding $\mathbf{R}=\mathbf{0}$. The superscript $\operatorname{Tr}$ of $G$ in Eq. (30) denotes transposing.

It can be shown (see Appendix B) that

$$
\sum_{\mathbf{R}} \exp \left(i \mathbf{k}_{\|} \cdot \mathbf{R}\right) \mathbf{H}_{l m 1}(\mathbf{r}-\mathbf{R})=\sum_{\mathbf{g}} \mathbf{B}_{l m 1}^{g \pm} \exp \left(i \mathbf{k}_{\alpha g}^{ \pm} \cdot \mathbf{r}\right)
$$

$$
\begin{aligned}
& \sum_{\mathbf{R}} \exp \left(i \mathbf{k}_{\|} \cdot \mathbf{R}\right) \mathbf{H}_{l m 2}(\mathbf{r}-\mathbf{R})=\sum_{\mathbf{g}} \mathbf{B}_{l m 2}^{g \pm} \exp \left(i \mathbf{k}_{\beta g}^{ \pm} \cdot \mathbf{r}\right), \\
& \sum_{\mathbf{R}} \exp \left(i \mathbf{k}_{\|} \cdot \mathbf{R}\right) \mathbf{H}_{l m 3}(\mathbf{r}-\mathbf{R})=\sum_{\mathbf{g}} \mathbf{B}_{l m 3}^{g \pm} \exp \left(i \mathbf{k}_{\beta g}^{ \pm} \cdot \mathbf{r}\right),
\end{aligned}
$$

where

$$
\begin{aligned}
& \mathbf{B}_{l m 1}^{g \pm}=\frac{2 \pi}{S} \frac{(-i)^{l-1}}{\alpha^{2}} \frac{Y_{l m}\left(\hat{\mathbf{k}}_{\alpha g}^{ \pm}\right)}{\left(\alpha^{2}-\left|\mathbf{k}_{\|}+\mathbf{g}\right|^{2}\right)^{1 / 2}} \mathbf{k}_{\alpha g}^{ \pm}, \\
& \mathbf{B}_{l m 2}^{g \pm}=\frac{2 \pi}{S} \frac{(-i)^{l+1}}{\beta \sqrt{l(l+1)}} \frac{1}{\left(\beta^{2}-\left|\mathbf{k}_{\|}+\mathbf{g}\right|^{2}\right)^{1 / 2}}\left\{\left[M_{l}^{m} Y_{l m+1}\left(\hat{\mathbf{k}}_{\beta g}^{ \pm}\right)\right.\right. \\
& \left.+N_{l}^{m} Y_{l m-1}\left(\hat{\mathbf{k}}_{\beta g}^{ \pm}\right)\right] \hat{\mathbf{x}}-i\left[M_{l}^{m} Y_{l m+1}\left(\hat{\mathbf{k}}_{\beta g}^{ \pm}\right)\right. \\
& \left.\left.-N_{l}^{m} Y_{l m-1}\left(\hat{\mathbf{k}}_{\beta g}^{ \pm}\right)\right] \hat{\mathbf{y}}+m Y_{l m}\left(\hat{\mathbf{k}}_{\beta g}^{ \pm}\right) \hat{\mathbf{z}}\right\}, \\
& \mathbf{B}_{l m 3}^{g \pm}=\frac{2 \pi}{S} \frac{(-i)^{l}}{\beta^{2} \sqrt{l(l+1)}} \frac{\mathbf{k}_{\beta g}^{ \pm}}{\left(\beta^{2}-\left|\mathbf{k}_{\|}+\mathbf{g}\right|^{2}\right)^{1 / 2}} \\
& \times\left\{\left[M_{l}^{m} Y_{l m+1}\left(\hat{\mathbf{k}}_{\beta g}^{ \pm}\right)+N_{l}^{m} Y_{l m-1}\left(\mathbf{k}_{\beta g}^{ \pm}\right)\right] \hat{\mathbf{x}}\right. \\
& -i\left[M_{l}^{m} Y_{l m+1}\left(\hat{\mathbf{k}}_{\beta g}^{ \pm}\right)-N_{l}^{m} Y_{l m-1}\left(\mathbf{k}_{\beta g}^{ \pm}\right)\right] \hat{\mathbf{y}} \\
& \left.+m Y_{l m}\left(\hat{\mathbf{k}}_{\beta g}^{ \pm}\right) \hat{\mathbf{z}}\right\} \text {, }
\end{aligned}
$$

and the sign + means $z>0$ and - means $z<0$. Thus,

$\mathbf{u}^{s c}(\mathbf{r})=\mathbf{u}_{\alpha}^{s c \pm}(\mathbf{r})+\mathbf{u}_{\beta}^{s c \pm}(\mathbf{r})$

$$
=\sum_{\mathbf{g}}\left[\mathbf{U}_{\alpha g}^{s c \pm} \exp \left(i \mathbf{k}_{\alpha g}^{ \pm} \cdot \mathbf{r}\right)+\mathbf{U}_{\beta g}^{s c \pm} \exp \left(i \mathbf{k}_{\beta g}^{ \pm} \cdot \mathbf{r}\right)\right],
$$

where

$$
\begin{gathered}
\mathbf{U}_{\alpha g}^{s c \pm}=\sum_{l m} b_{l m 1} \mathbf{B}_{l m 1}^{g \pm}, \\
\mathbf{U}_{\beta g}^{s c \pm}=\sum_{l m}\left(b_{l m 2} \mathbf{B}_{l m 2}^{g \pm}+b_{l m 3} \mathbf{B}_{l m 3}^{g \pm}\right) .
\end{gathered}
$$

By substituting Eq. (25) into Eq. (30), and then substituting the resulting expression into Eq. (35), we obtain

$$
\begin{aligned}
& \mathbf{U}_{\alpha g}^{s c s}=\sum_{s^{\prime} \mathbf{g}^{\prime}}\left(\mathbf{M}_{\alpha g \alpha g^{\prime}}^{s s^{\prime}} \cdot \mathbf{U}_{\alpha g^{\prime}}^{i n s^{\prime}}+\mathbf{M}_{\alpha g \beta g^{\prime}}^{s s^{\prime}} \cdot \mathbf{U}_{\beta g^{\prime}}^{i n s^{\prime}}\right), \\
& \mathbf{U}_{\beta g}^{s c s}=\sum_{s^{\prime} \mathbf{g}^{\prime}}\left(\mathbf{M}_{\beta g \alpha g^{\prime}}^{s s^{\prime}} \cdot \mathbf{U}_{\alpha g^{\prime}}^{i n s^{\prime}}+\mathbf{M}_{\beta g \beta g^{\prime}}^{s s^{\prime}} \cdot \mathbf{U}_{\beta g^{\prime}}^{i n s^{\prime}}\right),
\end{aligned}
$$

where $\mathbf{M}$ is defined as

$$
\mathbf{M}_{\alpha g \alpha g^{\prime}}^{s s^{\prime}}=\sum_{l m l^{\prime} m^{\prime}} \mathbf{B}_{l m 1}^{g s} Z_{l m 1 l^{\prime} m^{\prime} 1} \mathbf{A}_{l^{\prime} m^{\prime} 1}^{g^{\prime} s^{\prime}},
$$




$$
\begin{aligned}
& \mathbf{M}_{\alpha g \beta g^{\prime}}^{s s^{\prime}}=\sum_{l m l^{\prime} m^{\prime}}\left(\mathbf{B}_{l m 1}^{g s} Z_{l m 1 l^{\prime} m^{\prime} 2} \mathbf{A}_{l^{\prime} m^{\prime} 2}^{g^{\prime} s^{\prime}}\right. \\
& \left.+\mathbf{B}_{l m 1}^{g s} Z_{l m 1 l^{\prime} m^{\prime} 3} \mathbf{A}_{l^{\prime} m^{\prime} 3}^{g^{\prime} s^{\prime}}\right), \\
& \mathbf{M}_{\beta g \alpha g^{\prime}}^{s s^{\prime}}=\sum_{l m l^{\prime} m^{\prime}}\left(\mathbf{B}_{l m 2}^{g s} Z_{l m 2 l^{\prime} m^{\prime} 1} \mathbf{A}_{l^{\prime} m^{\prime} 1}^{g^{\prime} s^{\prime}}\right. \\
& \left.+\mathbf{B}_{l m 3}^{g s} Z_{l m 3 l^{\prime} m^{\prime} 1} \mathbf{A}_{l^{\prime} m^{\prime} 1}^{g^{\prime} s^{\prime}}\right), \\
& \mathbf{M}_{\beta g \beta g^{\prime}}^{s s^{\prime}}=\sum_{l m l^{\prime} m^{\prime}}\left(\mathbf{B}_{l m 2}^{g s} Z_{l m 2 l^{\prime} m^{\prime} 2} \mathbf{A}_{l^{\prime} m^{\prime} 2}^{g^{\prime} s^{\prime}}\right. \\
& +\mathbf{B}_{l m 3}^{g s} Z_{l m 3 l^{\prime} m^{\prime} 3} \mathbf{A}_{l^{\prime} m^{\prime} 3}^{g^{\prime} s^{\prime}}+\mathbf{B}_{l m 2}^{g s} Z_{l m 2 l^{\prime} m^{\prime} 3} \mathbf{A}_{l^{\prime} m^{\prime} 3}^{g^{\prime} s^{\prime}} \\
& +\mathbf{B}_{l m 3}^{g s} Z_{l m 3 l^{\prime} m^{\prime} 2} \mathbf{A}_{l^{\prime} m^{\prime} 2}^{g^{\prime} s^{\prime}} \text {. }
\end{aligned}
$$

Equation (36) may be expressed in matrix form:

$$
\begin{aligned}
& {\left[\begin{array}{c}
\mathbf{U}_{\alpha}^{s c+} \\
\mathbf{U}_{\beta}^{s c+}
\end{array}\right]=\left[\begin{array}{ll}
\mathbf{M}_{\alpha \alpha}^{++} & \mathbf{M}_{\alpha \beta}^{++} \\
\mathbf{M}_{\beta \alpha}^{++} & \mathbf{M}_{\beta \beta}^{++}
\end{array}\right]\left[\begin{array}{c}
\mathbf{U}_{\alpha}^{i n+} \\
\mathbf{U}_{\beta}^{i n+}
\end{array}\right]+\left[\begin{array}{ll}
\mathbf{M}_{\alpha \alpha}^{+-} & \mathbf{M}_{\alpha \beta}^{+-} \\
\mathbf{M}_{\beta \alpha}^{+-} & \mathbf{M}_{\beta \beta}^{+-}
\end{array}\right]\left[\begin{array}{c}
\mathbf{U}_{\alpha}^{i n-} \\
\mathbf{U}_{\beta}^{i n-}
\end{array}\right],} \\
& {\left[\begin{array}{c}
\mathbf{U}_{\alpha}^{s c-} \\
\mathbf{U}_{\beta}^{s c-}
\end{array}\right]=\left[\begin{array}{ll}
\mathbf{M}_{\alpha \alpha}^{-+} & \mathbf{M}_{\alpha \beta}^{-+} \\
\mathbf{M}_{\beta \alpha}^{-+} & \mathbf{M}_{\beta \beta}^{-+}
\end{array}\right]\left[\begin{array}{c}
\mathbf{U}_{\alpha}^{i n+} \\
\mathbf{U}_{\beta}^{i n+}
\end{array}\right]+\left[\begin{array}{ll}
\mathbf{M}_{\alpha \alpha}^{--} & \mathbf{M}_{\alpha \beta}^{--} \\
\mathbf{M}_{\beta \alpha}^{--} & \mathbf{M}_{\beta \beta}^{--}
\end{array}\right]\left[\begin{array}{c}
\mathbf{U}_{\alpha}^{i n-} \\
\mathbf{U}_{\beta}^{i n-}
\end{array}\right],}
\end{aligned}
$$

where $\mathbf{U}_{\kappa}^{s c s}$ and $\mathbf{U}_{\kappa}^{i n s}$ are column matrices and $\mathbf{M}_{\kappa \kappa^{\prime}}^{s s^{\prime}}$ are square matrices, defined as

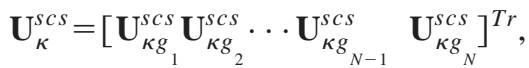

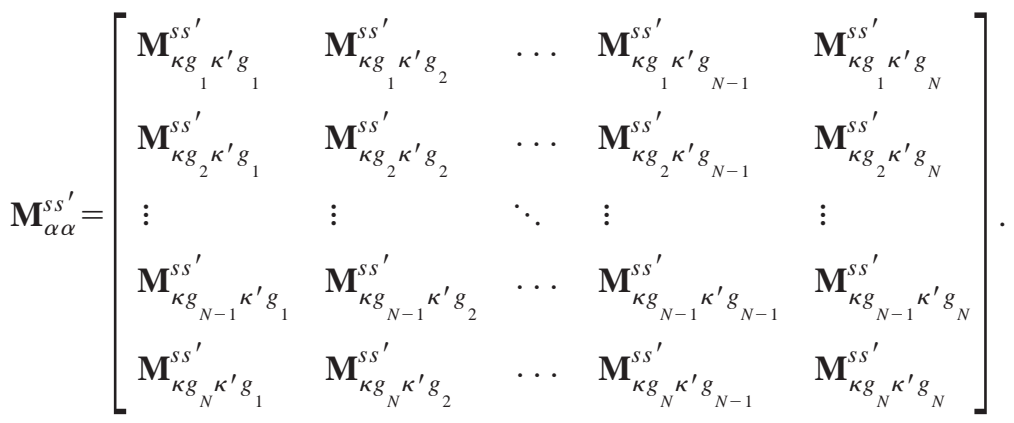

$\mathbf{M}_{\kappa \kappa^{\prime}}^{s s^{\prime}}$ gives the scattering of an incident plane elastic wave by a planar layer of periodically arranged scatterers.

\section{CALCULATION OF THE TRANSMISSION AND REFLECTION COEFFICIENTS}

To facilitate the derivation of the relevant formulas that follow, we write the displacement fields on both sides of the scattering plane in an alternative way. By naming the left side as side 1 and the right side as side 2, the wave traveling from the left to the right on side 1 and that along the opposite direction may be written as

$$
\begin{gathered}
{\left[\begin{array}{l}
\mathbf{U}_{\alpha}^{+}(1) \\
\mathbf{U}_{\beta}^{+}(1)
\end{array}\right]=\left[\begin{array}{c}
\mathbf{U}_{\alpha}^{i n+} \\
\mathbf{U}_{\beta}^{i n+}
\end{array}\right],} \\
{\left[\begin{array}{c}
\mathbf{U}_{\alpha}^{-}(1) \\
\mathbf{U}_{\beta}^{-}(1)
\end{array}\right]=\left[\begin{array}{c}
\mathbf{U}_{\alpha}^{s c-} \\
\mathbf{U}_{\beta}^{s c-}
\end{array}\right]+\left[\begin{array}{c}
\mathbf{U}_{\alpha}^{i n-} \\
\mathbf{U}_{\beta}^{i n-}
\end{array}\right] .}
\end{gathered}
$$

Similarly, at the right side, i.e., side 2 , we have

$$
\left[\begin{array}{c}
\mathbf{U}_{\alpha}^{+}(2) \\
\mathbf{U}_{\beta}^{+}(2)
\end{array}\right]=\left[\begin{array}{c}
\mathbf{U}_{\alpha}^{i n+} \\
\mathbf{U}_{\beta}^{i n+}
\end{array}\right]+\left[\begin{array}{c}
\mathbf{U}_{\alpha}^{s c+} \\
\mathbf{U}_{\beta}^{s c+}
\end{array}\right],
$$

$$
\left[\begin{array}{c}
\mathbf{U}_{\alpha}^{-}(2) \\
\mathbf{U}_{\beta}^{-}(2)
\end{array}\right]=\left[\begin{array}{c}
\mathbf{U}_{\alpha}^{i n-} \\
\mathbf{U}_{\beta}^{i n-}
\end{array}\right] .
$$

Substituting Eq. (38) into Eq. (41) and Eq. (42), we obtain

$$
\begin{aligned}
{\left[\begin{array}{l}
\mathbf{U}_{\alpha}^{+}(2) \\
\mathbf{U}_{\beta}^{+}(2)
\end{array}\right]=} & {\left[\begin{array}{ll}
\mathbf{I}+\mathbf{M}_{\alpha \alpha}^{++} & \mathbf{M}_{\alpha \beta}^{++} \\
\mathbf{M}_{\beta \alpha}^{++} & \mathbf{I}+\mathbf{M}_{\beta \beta}^{++}
\end{array}\right]\left[\begin{array}{l}
\mathbf{U}_{\alpha}^{+}(1) \\
\mathbf{U}_{\beta}^{+}(1)
\end{array}\right] } \\
& +\left[\begin{array}{ll}
\mathbf{M}_{\alpha \alpha}^{+-} & \mathbf{M}_{\alpha \beta}^{+-} \\
\mathbf{M}_{\beta \alpha}^{+-} & \mathbf{M}_{\beta \beta}^{+-}
\end{array}\right]\left[\begin{array}{l}
\mathbf{U}_{\alpha}^{-}(2) \\
\mathbf{U}_{\beta}^{-}(2)
\end{array}\right], \\
{\left[\begin{array}{c}
\mathbf{U}_{\alpha}^{-}(1) \\
\mathbf{U}_{\beta}^{-}(1)
\end{array}\right]=} & {\left[\begin{array}{ll}
\mathbf{M}_{\alpha \alpha}^{-+} & \mathbf{M}_{\alpha \beta}^{-+} \\
\mathbf{M}_{\beta \alpha}^{-+} & \mathbf{M}_{\beta \beta}^{-+}
\end{array}\right]\left[\begin{array}{l}
\mathbf{U}_{\alpha}^{+}(1) \\
\mathbf{U}_{\beta}^{+}(1)
\end{array}\right] } \\
& +\left[\begin{array}{ll}
\mathbf{I}+\mathbf{M}_{\alpha \alpha}^{--} & \mathbf{M}_{\alpha \beta}^{--} \\
\mathbf{M}_{\beta \alpha}^{--} & \mathbf{I}+\mathbf{M}_{\beta \beta}^{--}
\end{array}\right]\left[\begin{array}{l}
\mathbf{U}_{\alpha}^{-}(2) \\
\mathbf{U}_{\beta}^{-}(2)
\end{array}\right] .
\end{aligned}
$$

One should note that all the plane-wave expansions, including the incident and scattered waves, are referred to the central scatterer in the plane. If we shift the center of expansion by $-\mathbf{a}_{3} / 2$ for waves on side 1 and by $\mathbf{a}_{3} / 2$ for waves on side 2 , where $\mathbf{a}_{3}$ is the translation vector of the two-dimensional plane in forming a three-dimensional crystal, then 


$$
\begin{aligned}
{\left[\begin{array}{c}
\mathbf{U}_{\alpha}^{+}(2) \\
\mathbf{U}_{\beta}^{+}(2)
\end{array}\right]=} & {\left[\begin{array}{ll}
\mathbf{Q}_{\alpha \alpha}^{++} & \mathbf{Q}_{\alpha \beta}^{++} \\
\mathbf{Q}_{\beta \alpha}^{++} & \mathbf{Q}_{\beta \beta}^{++}
\end{array}\right]\left[\begin{array}{l}
\mathbf{U}_{\alpha}^{+}(1) \\
\mathbf{U}_{\beta}^{+}(1)
\end{array}\right]+\left[\begin{array}{ll}
\mathbf{Q}_{\alpha \alpha}^{+-} & \mathbf{Q}_{\alpha \beta}^{+-} \\
\mathbf{Q}_{\beta \alpha}^{+-} & \mathbf{Q}_{\beta \beta}^{+-}
\end{array}\right] } \\
& \times\left[\begin{array}{l}
\mathbf{U}_{\alpha}^{-}(2) \\
\mathbf{U}_{\beta}^{-}(2)
\end{array}\right], \\
{\left[\begin{array}{c}
\mathbf{U}_{\alpha}^{-}(1) \\
\mathbf{U}_{\beta}^{-}(1)
\end{array}\right]=} & {\left[\begin{array}{ll}
\mathbf{Q}_{\alpha \alpha}^{-+} & \mathbf{Q}_{\alpha \beta}^{-+} \\
\mathbf{Q}_{\beta \alpha}^{-+} & \mathbf{Q}_{\beta \beta}^{-+}
\end{array}\right]\left[\begin{array}{l}
\mathbf{U}_{\alpha}^{+}(1) \\
\mathbf{U}_{\beta}^{+}(1)
\end{array}\right]+\left[\begin{array}{ll}
\mathbf{Q}_{\alpha \alpha}^{--} & \mathbf{Q}_{\alpha \beta}^{--} \\
\mathbf{Q}_{\beta \alpha}^{--} & \mathbf{Q}_{\beta \beta}^{--}
\end{array}\right] } \\
& \times\left[\begin{array}{l}
\mathbf{U}_{\alpha}^{-}(2) \\
\mathbf{U}_{\beta}^{-}(2)
\end{array}\right],
\end{aligned}
$$

where

$$
\mathbf{Q}_{\kappa \kappa^{\prime}}^{s s^{\prime}}=\phi_{\kappa}^{s} \phi_{\kappa^{\prime}}^{s^{\prime}} \delta_{\kappa \kappa^{\prime}} \delta_{s s^{\prime}}+\phi_{\kappa}^{s} M_{\kappa \kappa^{\prime}}^{s s^{\prime}} \phi_{\kappa^{\prime}}^{s^{\prime}},
$$

with matrices $\phi_{\kappa}^{s}$ defined as

$$
\phi_{\kappa}^{s}=\left[\begin{array}{lll}
\exp \left(s i \mathbf{k}_{\kappa g_{1}}^{s} \cdot \mathbf{a}_{3} / 2\right) & & \\
& \ddots & \\
& & \exp \left(s i \mathbf{k}_{\kappa g_{N}}^{s} \cdot \mathbf{a}_{3} / 2\right)
\end{array}\right] .
$$

Once the $\mathbf{Q}$ matrices for one scattering plane are determined, one can easily obtain the $\mathbf{Q}$ matrices of a slab with two scattering planes. ${ }^{11}$ The procedure can be repeated to obtain the $\mathbf{Q}$ matrices for a slab with $2^{n}$ scattering planes, with $n$ being an arbitrary integer. The proper combination of these slabs enables us to obtain the $\mathbf{Q}$ matrices for a slab with any number of scattering planes.

Once the $\mathbf{Q}$ matrices for a slab are obtained, we can completely determine the transmitted and reflected waves from Eq. (44), given the incident waves. Since the flux for a longitudinal elastic wave is given by $(\lambda+2 \mu) \omega\left(\mathbf{U}_{\alpha g} \cdot \mathbf{U}_{\alpha g}^{*}\right) \mathbf{k}_{\alpha g}$ and that for a transverse elastic wave is given by $\mu \omega\left(\mathbf{U}_{\beta g} \cdot \mathbf{U}_{\beta g}^{*}\right) \mathbf{k}_{\beta g}$, the transmittance $\mathcal{T}$ and reflectance $\mathcal{R}$ for elastic waves from a slab (with the normal direction along the $z$ axis) is thus given by

$$
\mathcal{T}(\mathcal{R})=\frac{\sum_{\mathbf{g}}\left\{(\lambda+2 \mu) \mathbf{U}_{\alpha g}^{t r n(r e f)} \cdot \mathbf{U}_{\alpha g}^{t r n(r e f) *} k_{\alpha g z}^{+}+\mu \mathbf{U}_{\beta g}^{t r n(r e f)} \cdot \mathbf{U}_{\beta g}^{t r n(r e f) *} k_{\beta g z}^{+}\right\}}{\sum_{\mathbf{g}}\left\{(\lambda+2 \mu) \mathbf{U}_{\alpha g}^{i n c} \cdot \mathbf{U}_{\alpha g}^{i n c *} k_{\alpha g z}^{+}+\mu \mathbf{U}_{\beta g}^{i n c} \cdot \mathbf{U}_{\beta g}^{i n c *} k_{\beta g z}^{+}\right\}} .
$$

The requirement for energy conservation implies that the absorbance $\xi$ for a system with loss is given by

$$
\xi=1-\mathcal{T}-\mathcal{R} .
$$

\section{NUMERICAL RESULTS AND DISCUSSION}

We have performed band structure calculations for gold spheres embedded in a silicon matrix, arranged in various periodic structures. It has been shown that for the fcc structure there can be electromagnetic as well as elastic band gaps. ${ }^{1,4}$ The middle panel of Fig. 1 is the fcc band structure for the $\mathrm{Au} / \mathrm{Si}$ composite with a filling ratio of $10 \%$, which was reported to have the largest elastic stop band for this system. ${ }^{4}$ The material parameters used in the calculation are $\rho=19.5 \mathrm{~g} / \mathrm{cm}^{3}, c_{l}=3.36 \mathrm{~km} / \mathrm{s}, c_{l} / c_{t}=2.71$ for $\mathrm{Au}$, and $\rho$ $=2.33 \mathrm{~g} / \mathrm{cm}^{3}, c_{l}=8.95 \mathrm{~km} / \mathrm{s}, c_{l} / c_{t}=1.67$ for Si. The cutoff angular momentum is set at $l_{\max }=3$. Convergence was checked at selected points in the Brillouin zone by using $l_{\max }=5$. Only changes on the order of $0.1 \%$ were found up to the maximum frequency shown in the figure, indicating excellent convergence. For higher filling fractions of $\mathrm{Au}$ spheres, a larger $l_{\max }$ setting is required. However, excellent convergence is always obtained $\left(l_{\max }=7\right.$ is good enough for filling fractions close to close packed). The calculation is also reasonably fast. For the case of $10 \%$ filling ratio, only 40 min on a Pentium II $400 \mathrm{MHz}$ machine (with 128 megabyte memory) is required to complete the band structure cal- culation. For $l_{\max }=5$, which is sufficient for a filling ratio of $60 \%$, only $80 \mathrm{~min}$ is required. Our results are noted to coincide with those of Ref. 4 for the lower frequencies. However, at higher frequencies there are some minor differences. As we can see, there is a small absolute band gap at frequencies just above 0.6 (in units of $2 \pi c_{t} / a$ ), first reported by Econo-

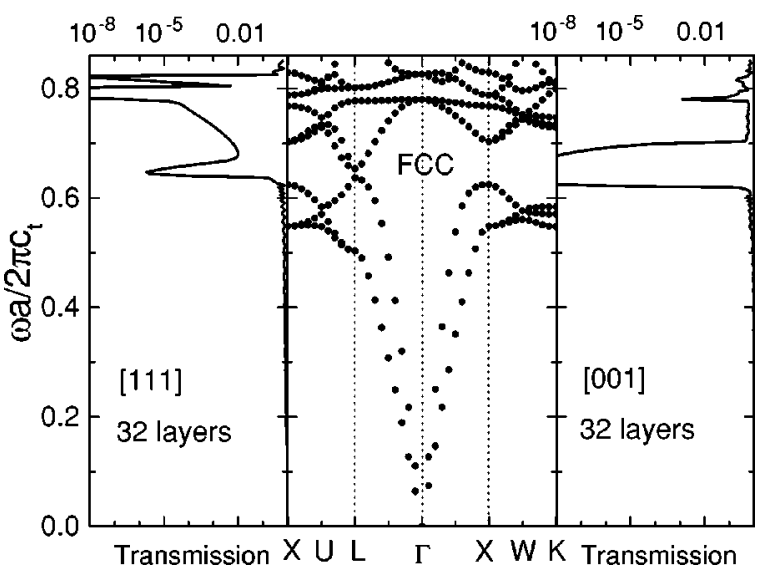

FIG. 1. Band structure and amplitude transmission coefficient of elastic waves propagating in a fcc structure formed with gold spheres embedded in a silicon matrix. The filling ratio of spheres is 10\%. (a) Right panel: transmission amplitude through 32 layers along the [001] direction. (b) Middle panel: band structure of this system. (c) Left panel: same as (a), but along the [111] direction. 


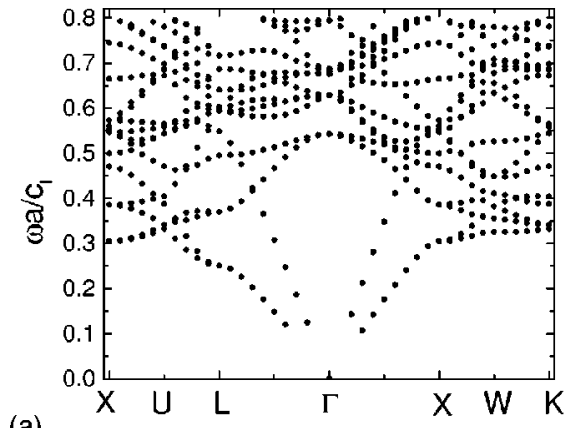

(a)

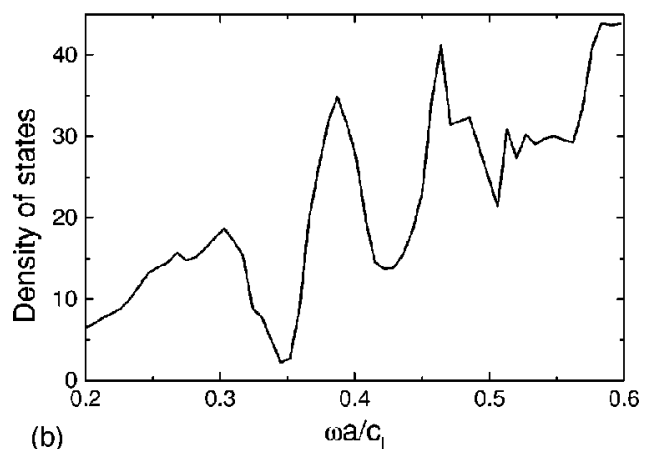

FIG. 2. (a) The band structure of elastic waves propagating in an isotropic solid with spherical voids arranged in a fcc structure. The filling ratio is $34 \%$. The velocity ratio of the transverse wave to the longitudinal wave in the matrix medium is 0.4 . (b) The corresponding density of states.

mou and co-workers. ${ }^{4}$ The transmission calculation for elastic waves propagating along the (001) and (111) directions in this structure with a thickness of 32 layers are shown in the left and right panels of Fig. 1. Excellent agreement is found with the band structure. In particular, we note that the sizable directional stop band in the transmission along (001) centered at about 0.65 units coincides with the corresponding directional gap along the $\Gamma-X$ direction in the band structure. In the transmission along (111), we observe a narrow stop band at about 0.65 units, corresponding to the small gap at the $L$ point in the band structure at the same frequency.

In the second set of examples, we calculate the elastic wave band structure for a system involving just one solid medium, with periodically arranged spherical voids. Unlike other heterogeneous elastic systems, this system involves just two intrinsic parameters - the filling ratio $\phi$ of the voids, and the ratio $c_{t} / c_{l}$ of the longitudinal to the transverse sound velocities in the host medium. The small number of parameters means that we can afford to carry out a more complete search for elastic wave band gaps in this system. Figure 2(a) shows the band structure of elastic waves in the fcc structure, for a typical parameter set of $\phi=0.34, c_{t} / c_{l}=0.4$. Similar band structures have been calculated for bcc, hcp, and diamond structures, with void fraction varying from $10 \%$ to $66 \%$. We do not find a complete gap in this system within the range of our search. However, deep dips in the density of states $^{18}$ do exist, as shown in Fig. 2(b). By comparing the density of states with the band structure, we observe that at the first dip frequency, transverse waves are almost forbidden and only the longitudinal modes propagate. Thus in this case the absence of the transverse modes accounts for the low density of states.

\section{EXPERIMENTAL RESULTS AND DISCUSSION}

To further examine and test the predictions of our MST approach, we have compared our theoretical calculations with ultrasonic experiments on a hexagonal-close-packed (hcp) array of stainless steel beads immersed in water. In this case the water matrix supports only longitudinal waves, although the full elastic behavior of the steel scatterers must, of course, be included. The samples were constructed using steel balls that were extremely monodisperse and accurately spherical in shape, with a diameter of $0.8014 \pm 0.0005 \mathrm{~mm}$ and a sphericity of $0.25 \mu \mathrm{m}$. The hcp crystal was prepared by placing the beads very carefully by hand in an acrylite cell having hexagonal sidewalls which were accurately positioned to force the first layer of beads to form a defect-free triangular lattice. Subsequent layers were added in an $A B A B A B \ldots$ sequence, in which each layer was constrained in a triangular arrangement by the beads underneath, thus forming a hcp lattice with $c$ axis perpendicular to the layers. Two slab-shaped samples were prepared, one having five and the other ten layers, with each layer containing a sufficiently large number of beads (approximately 6000) that boundary reflections at the perimeter of each layer could be neglected. The choice of steel beads (with longitudinal and shear velocities $c_{l}=6.01 \mathrm{~km} / \mathrm{s}$ and $c_{t}=3.23 \mathrm{~km} / \mathrm{s}$, and density $\left.\rho=7.67 \times 10^{3} \mathrm{~kg} / \mathrm{m}^{3}\right)$ and a water matrix $\left(c_{l}\right.$ $\left.=1.49 \mathrm{~km} / \mathrm{s}, \rho=1.0 \times 10^{3} \mathrm{~kg} / \mathrm{m}^{3}\right)$ ensured that high contrast was achieved in these ultrasonic experiments on account of the large difference in their elastic properties.

We used pulsed experiments to directly measure the ultrasonic wave field that was transmitted parallel to the $c$ axis of the crystals, allowing us to determine both the dispersion curve and amplitude transmission coefficient along this direction. These measurements were accomplished by placing the samples in a water tank between two planar immersion transducers that were oriented so that the direction of propagation was vertical. Thus there was no need for a top wall on the sample, simplifying the boundary conditions. The bottom supporting wall was made sufficiently thick that no multiple reflections in the wall could arrive at the lower sample face until after the initial transmitted pulse through the sample had decayed to below the detection noise threshold. To ensure that the incident pulse was a good approximation to a plane wave, the sample was placed in the far field of the generating transducer, positioned on the bottom of the tank. The receiving transducer was $25 \mathrm{~mm}$ in diameter and was placed sufficiently far above the sample to avoid complications due to multiple reflections between sample and receiver. A digital oscilloscope was used to record the signalaveraged time-domain wave forms of the transmitted field both with and without the sample in place. Thus it was straightforward to account for the presence of the support wall in making accurate phase and amplitude measurements of the field propagating through the sample. A typical input pulse and the corresponding transmitted field through the ten-layer sample are shown in Figs. 3(a) and 3(b); note the marked increase in duration of the transmitted pulse, and the modulation of its shape, due to the interference effects caused by Bragg scattering within the crystal. Four pairs of broadband immersion transducers were used to measure the transmitted field over a continuous range of frequencies from 


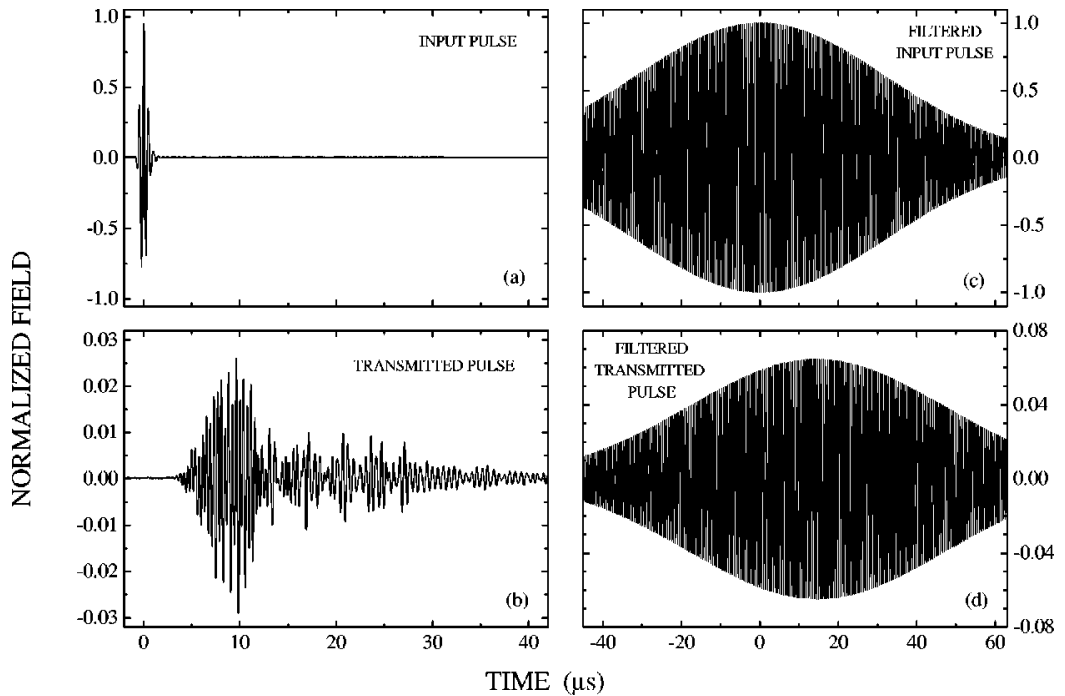

FIG. 3. Typical input (a) and transmitted (b) ultrasonic pulses through a ten-layer hcp crystal of stainless steel beads in water. The input pulse shown in (a) represents the pulse that has traveled through the support layer and is incident at the interface between the support layer and the crystalline sample. The results obtained by digitally filtering the pulses at a central frequency of 2.0 $\mathrm{MHz}$ and a bandwidth of $0.1 \mathrm{MHz}$ are shown in (c) and (d), allowing the phase velocity to be determined by measuring the phase delay. For both the unfiltered and filtered pulses, the data have been normalized to center the input pulses at $t$ $=0 \mu \mathrm{s}$, and to scale the vertical axes so that the peak amplitude of the input pulses is unity. below $0.5 \mathrm{MHz}$ to over $4 \mathrm{MHz}$, corresponding to the frequencies at which the lowest band gaps are expected in this crystal.

To determine the band structure, we first measured the phase velocity $v_{p}$ by digitally filtering the transmitted wave forms using a narrow-band Gaussian filter centered at frequency $f=\omega / 2 \pi$ and determining the phase delay between the filtered input and transmitted pulses at this frequency. The process was then repeated over the entire frequency range spanned by the transducers. The filter bandwidth was chosen to be sufficiently narrow that the filtered pulses extended over a long enough time to incorporate the contributions to the net phase from all multiply reflected waves in the sample. Typical examples of the digitally filtered pulses are shown in Figs. 3(c) and 3(d), for a central frequency of 2.0 $\mathrm{MHz}$ and a bandwidth of $0.01 \mathrm{MHz}$. The phase velocity was then determined from the ratio of the sample thickness to the measured phase delay between the input and transmitted pulses. Since the measurements were performed for two sample thicknesses, uncertainties in the phase shift of multiples of $2 \pi$ could be unambiguously eliminated. Note that this method of determining the phase delay is equivalent to measuring the phase difference directly from the fast Fourier transforms of the input and transmitted pulses ${ }^{19}$ however, our digital filtering method allows the phase delay to be readily visualized in the time domain, where it can be regarded as a simple generalization of conventional pulsed ultrasonic techniques. These measurements of the phase velocity directly give the dispersion curve, $\omega$ versus $k=\omega / v_{p}$, in the extended zone scheme. The band structure in the usual reduced zone scheme was then obtained by subtracting appropriate multiples of the reciprocal lattice vector $\mathbf{G}_{001}$ $=2 \pi / c \hat{\mathbf{k}}$ from the wave vector in the extended zone scheme and making use of the symmetry of the dispersion curve about $\pm \hat{\mathbf{k}}$, where $\hat{\mathbf{k}}$ denotes a unit vector parallel to the $c$ axis. Our experimental results for the ten-layer sample are compared with the theoretical predictions of the MST theory in Fig. 4(a), where the solid squares connected by dashed curves represent the experimental data, and the solid curves represent the theoretical calculations. Overall, the agreement between theory and experiment is very good over this range of frequencies. However, at higher frequencies (not shown) the agreement is not as good, probably reflecting small imperfections in the crystal that become more important at shorter wavelengths. Other possible reasons for disagreement could be the fact that the experiment was performed on a ten-layer sample, while the theory is for an infinite medium, and also the possible influence of the support layer in the experiments.
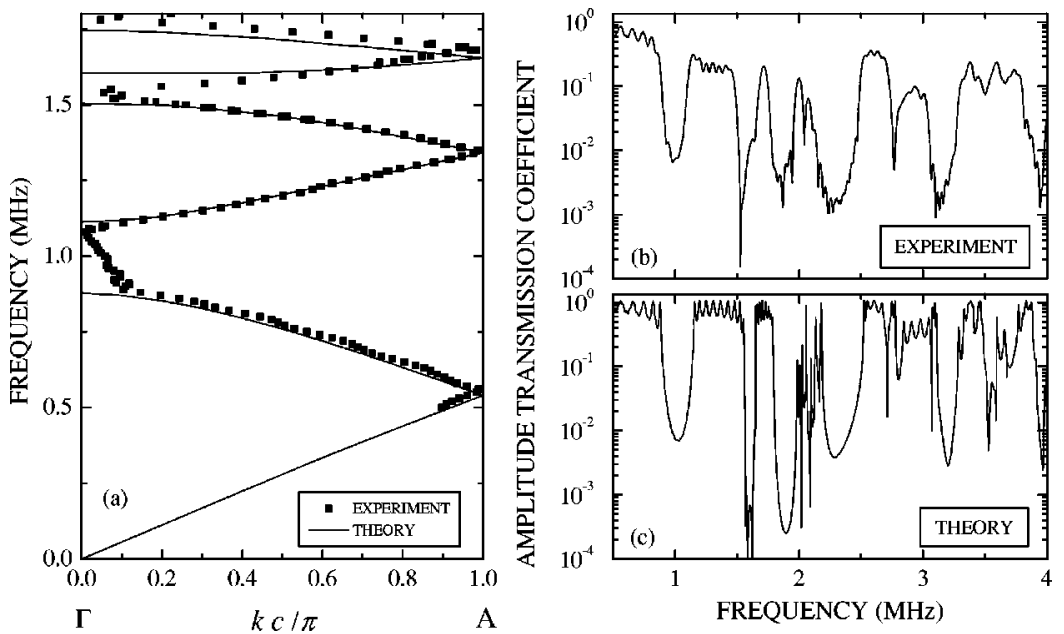

FIG. 4. Band structure and amplitude transmission coefficient of ultrasound waves propagating along the [001] direction of a hcp structure consisting of stainless steel balls immersed in water. (a) Band structure. (b) and (c) Amplitude transmission coefficient through the ten-layer sample. The upper panel gives the experimental results (b), while the lower panel shows the theoretical calculations (c). Excellent overall agreement is seen. 
By taking the ratio of the fast Fourier transforms of the transmitted and input pulses, we also measured the frequency dependence of the amplitude transmission coefficient. Figure 4(b) shows the experimental results for the ten-layer sample, where we plot the frequency dependence of the transmitted amplitude through the crystal sample and its support layer, normalized by the amplitude of the pulse transmitted through the support layer only. Thus attenuation in the support layer did not add a misleading additional contribution to the measured transmission. These results are compared with our theoretical calculations in Fig. 4(c). To ensure that the theory and experiment are normalized in the same way, boundary reflections at the input and output faces of the crystal samples were also included in the calculations, using the known phase velocities and densities of the support wall and surrounding water. However, the effects of multiple reflections in the support layer were eliminated from the calculations, since they were not measured in our time-resolved pulsed experiments. ${ }^{20}$ Comparison of Figs. 4(b) and 4(c) shows that excellent correspondence between theory and experiment is found in the positions of the minima and maxima in the transmitted amplitude. There is also excellent correspondence between the positions of the transmission minima and the gaps in the dispersion curve. The small evenly spaced oscillations in the transmitted amplitude arise from multiple reflections in the crystal slab; conclusive evidence for this comes both from the fact that these oscillations were less closely spaced in the thinner sample (not shown) and from a calculation of this interference effect based on the measured frequency dependence of the phase velocity in the sample. While the overall structure of the measured transmission coefficient is well captured by the theoretical calculations, the magnitude of the measured transmission is consistently lower, the difference becoming more pronounced at higher frequencies. This difference arises from absorption in the sample, most likely due to viscous losses at the water/ solid interfaces, an effect that has not been included in the present calculations.

\section{CONCLUDING REMARKS}

In summary, we have extended multiple-scattering theory for elastic waves and demonstrated its application to two sets of examples. In the first example, we show the applicability of our theories through comparison of the band structure and transmission calculations. In another example, we find no elastic wave band gap in the system of an isotropic solid filled with periodically arranged voids. The band structure and transmission calculations for a hcp array of steel balls immersed in water show excellent agreement with experiment.

\section{ACKNOWLEDGMENTS}

This work is supported in part by the Hong Kong RGC Grant No. HKUST685/96P, HKUST6136/97P, and HKUST6145/99P, and by NSERC of Canada. We thank Dr. W. Y. Zhang, Dr Z. F. Lin and Dr. Z. L. Wang for their assistance.

Note added. Recently noted that a paper by Kafesaji and Economou on the acoustic wave KKR approach appeared, ${ }^{21}$ using the Green's function formalism. In contrast, our work uses a multiple-scattering approach, and the code is suitable for both acoustic and elastic waves. In addition, the formalism for both transmission and reflection calculations has been formulated and implemented, thus facilitating comparison with experiment. We note also that Psarobas, Stefanou, and Modinos ${ }^{22}$ have formulated a layer KKR approach similar to our formulation in Sec. IV.

\section{APPENDIX A}

In this appendix, we prove Eqs. (9) and (10), where the vector structure constant $G_{l m \sigma l^{\prime} m^{\prime} \sigma^{\prime}}$ is defined by the relation

$$
\mathbf{H}_{l m \sigma}(\mathbf{r}+\mathbf{R})=\sum_{l^{\prime} m^{\prime} \sigma^{\prime}} G_{l m \sigma l^{\prime} m^{\prime} \sigma^{\prime}}(\mathbf{R}) \mathbf{J}_{l^{\prime} m^{\prime} \sigma^{\prime}}(\mathbf{r})
$$

It is known that

$$
\begin{aligned}
h_{l}(\kappa \mid \mathbf{r} & +\mathbf{R} \mid) Y_{l m}(\widehat{\mathbf{r}+\mathbf{R}}) \\
= & \sum_{l^{\prime} m^{\prime}} j_{l^{\prime}}(\kappa r) Y_{l^{\prime} m^{\prime}}(\hat{\mathbf{r}}) \sum_{l^{\prime \prime}} 4 \pi i^{l^{\prime}+l^{\prime \prime}-l} C_{l^{\prime} m^{\prime} l^{\prime \prime} m-m^{\prime}}^{l m} \\
& \times h_{l^{\prime \prime}}(\kappa R) Y_{l^{\prime \prime} m-m^{\prime}}(\hat{\mathbf{R}}) \\
= & \sum_{l^{\prime} m^{\prime}} X_{l m l^{\prime} m^{\prime}}^{\kappa}(\mathbf{R}) j_{l^{\prime}}(\kappa r) Y_{l^{\prime} m^{\prime}}(\hat{\mathbf{r}})
\end{aligned}
$$

Thus, according to the definition of $\mathbf{H}_{l m \sigma}$,

$$
\begin{aligned}
\mathbf{H}_{l m 1}(\mathbf{r}+\mathbf{R}) & =\frac{1}{\alpha} \boldsymbol{\nabla}\left[h_{l}(\alpha|\mathbf{r}+\mathbf{R}|) Y_{l m}(\widehat{\mathbf{r}+\mathbf{R}})\right] \\
& =\sum_{l^{\prime} m^{\prime}} X_{l m l^{\prime} m^{\prime}}^{\alpha}(\mathbf{R}) \frac{1}{\alpha} \nabla\left[j_{l^{\prime}}(\alpha r) Y_{l^{\prime} m^{\prime}}(\hat{\mathbf{r}})\right] \\
& =\sum_{l^{\prime} m^{\prime}} X_{l m l^{\prime} m^{\prime}}^{\alpha}(\mathbf{R}) \mathbf{J}_{l^{\prime} m^{\prime} 1}(\mathbf{r}) \\
& =\sum_{l^{\prime} m^{\prime} \sigma^{\prime}} X_{l m l^{\prime} m^{\prime}}^{\alpha}(\mathbf{R}) \delta_{1 \sigma^{\prime}} \mathbf{J}_{l^{\prime} m^{\prime} \sigma^{\prime}}(\mathbf{r}) .
\end{aligned}
$$

Comparison of Eq. (A1) with the last line of Eq. (A3) gives

$$
G_{l m 1 l^{\prime} m^{\prime} \sigma^{\prime}}(\mathbf{R})=X_{l m l^{\prime} m^{\prime}}^{\alpha}(\mathbf{R}) \delta_{1 \sigma^{\prime}} .
$$

Now we turn to $\mathbf{H}_{l m 2}(\mathbf{r}+\mathbf{R})$. From the definition Eq. (4) of $\mathbf{H}_{l m 2}(\mathbf{r})$, we get

$$
\mathbf{H}_{l m 2}(\mathbf{r})=-\frac{1}{\sqrt{l(l+1)}} h_{l}(\beta r) \mathbf{r} \times \nabla Y_{l m}(\hat{\mathbf{r}}),
$$

from which we can write

$$
\mathbf{H}_{l m 2}(\mathbf{r})=-i h_{l}(\beta r) \sum_{\mu} c(l 1 l, m-\mu \mu) Y_{l m-\mu}(\hat{\mathbf{r}}) \hat{\boldsymbol{\xi}}_{\mu},
$$

where 


$$
\begin{gathered}
\hat{\boldsymbol{\xi}}_{1}=-\frac{1}{\sqrt{2}}(\hat{\mathbf{x}}+i \hat{\mathbf{y}}), \\
\hat{\boldsymbol{\xi}}_{0}=\hat{\mathbf{z}}, \\
\hat{\boldsymbol{\xi}}_{-1}=\frac{1}{\sqrt{2}}(\hat{\mathbf{x}}-i \hat{\mathbf{y}}),
\end{gathered}
$$

and $c(l 1 l, m-\mu \mu)$ are the Clebsch-Gordan coefficients. Thus

$$
\begin{aligned}
\mathbf{H}_{l m 2}(\mathbf{r}+\mathbf{R})= & -i \sum_{\mu} c(l 1 l, m-\mu \mu) h_{l}(\beta|\mathbf{r}+\mathbf{R}|) Y_{l m-\mu} \\
& \times(\widehat{\mathbf{r}+\mathbf{R}}) \hat{\boldsymbol{\xi}}_{\mu} .
\end{aligned}
$$

Substituting Eq. (A2) into the above equation and following the derivation by Wang et al., ${ }^{3}$ we arrive at

$$
\begin{aligned}
\mathbf{H}_{l m 2}(\mathbf{r}+\mathbf{R})= & \sum_{\mu l^{\prime} m^{\prime}} c(l 1 l, m-\mu \mu) \\
& \times\left[X _ { l m - \mu l ^ { \prime } m ^ { \prime } - \mu } ^ { \beta } ( \mathbf { R } ) c \left(l^{\prime} 1 l^{\prime}, m^{\prime}\right.\right. \\
& -\mu \mu) \mathbf{J}_{l^{\prime} m^{\prime} 2}(\mathbf{r}) \\
& -i\left(\frac{2 l^{\prime}+1}{l^{\prime}+1}\right)^{1 / 2} X_{l m-\mu l^{\prime}-1 m^{\prime}-\mu}^{\beta}(\mathbf{R}) \\
& \left.\times c\left(l^{\prime}-11 l^{\prime}, m^{\prime}-\mu \mu\right) \mathbf{J}_{l^{\prime} m^{\prime} 3}(\mathbf{r})\right] .
\end{aligned}
$$

From Eq. (A9) we obtain

$$
\begin{aligned}
G_{l m 2 l^{\prime} m^{\prime} 2}(\mathbf{R})= & \sum_{\mu} c(l 1 l, m-\mu \mu) \\
& \times X_{l m-\mu l^{\prime} m^{\prime}-\mu}^{\beta}(\mathbf{R}) c\left(l^{\prime} 1 l^{\prime}, m^{\prime}-\mu \mu\right), \\
G_{l m 2 l^{\prime} m^{\prime} 3}(\mathbf{R})= & -i\left(\frac{2 l^{\prime}+1}{l^{\prime}+1}\right)^{1 / 2} \sum_{\mu} c(l 1 l, m-\mu \mu) \\
& \times X_{l m-\mu l^{\prime}-1 m^{\prime}-\mu}^{\beta}(\mathbf{R}) \\
& \times c\left(l^{\prime}-11 l^{\prime}, m^{\prime}-\mu \mu\right) .
\end{aligned}
$$

To get the expression for $\mathbf{H}_{l m 3}(\mathbf{r}+\mathbf{R})$, we use $(1 / \beta) \boldsymbol{\nabla} \times$ to act on Eq. (A9), leading to

$$
\begin{aligned}
\mathbf{H}_{l m 3}(\mathbf{r}+\mathbf{R})= & \sum_{\mu l^{\prime} m^{\prime}} c(l 1 l, m-\mu \mu) \\
& \times\left[-i\left(\frac{2 l^{\prime}+1}{l^{\prime}+1}\right)^{1 / 2} X_{l m-\mu l^{\prime}-1 m^{\prime}-\mu}^{\beta}(\mathbf{R}) c\left(l^{\prime}\right.\right. \\
& \left.-11 l^{\prime}, m^{\prime}-\mu \mu\right) \mathbf{J}_{l^{\prime} m^{\prime} 2}(\mathbf{r})+X_{l m-\mu l^{\prime} m^{\prime}-\mu}^{\beta}(\mathbf{R}) \\
& \left.\times c\left(l^{\prime} 1 l^{\prime}, m^{\prime}-\mu \mu\right) \mathbf{J}_{l^{\prime} m^{\prime} 3}(\mathbf{r})\right] .
\end{aligned}
$$

From this we get

$$
\begin{aligned}
G_{l m 3 l^{\prime} m^{\prime} 2}(\mathbf{R})= & -i\left(\frac{2 l^{\prime}+1}{l^{\prime}+1}\right)^{1 / 2} \sum_{\mu} c(l 1 l, m \\
& -\mu \mu) X_{l m-\mu l^{\prime}-1 m^{\prime}-\mu}^{\beta}(\mathbf{R}) \\
& \times c\left(l^{\prime}-11 l^{\prime}, m^{\prime}-\mu \mu\right),
\end{aligned}
$$

$$
\begin{aligned}
G_{l m 3 l^{\prime} m^{\prime} 3}(\mathbf{R})= & \sum_{\mu} c(l 1 l, m-\mu \mu) \\
& \times X_{l m-\mu l^{\prime} m^{\prime}-\mu}^{\beta}(\mathbf{R}) c\left(l^{\prime} 1 l^{\prime}, m^{\prime}-\mu \mu\right) .
\end{aligned}
$$

\section{APPENDIX B}

In this appendix, we prove Eqs. (25) and (26) and Eqs. (32) and (33). First, we introduce an equation that can be obtained from the definitions of $\mathbf{J}_{l m 2}(\mathbf{r})$ and $\mathbf{H}_{l m 2}(\mathbf{r})$, and Eq. (A6):

$$
\begin{aligned}
\mathbf{J}_{l m 2}(\mathbf{r})= & -\frac{i}{\sqrt{l(l+1)}} j_{l}(\beta r)\left\{\left[M_{l}^{m} Y_{l m+1}(\hat{\mathbf{r}})\right.\right. \\
& \left.+N_{l}^{m} Y_{l m-1}(\hat{\mathbf{r}})\right] \hat{\mathbf{x}}-i\left[M_{l}^{m} Y_{l m+1}(\hat{\mathbf{r}})\right. \\
& \left.\left.-N_{l}^{m} Y_{l m-1}(\hat{\mathbf{r}})\right] \hat{\mathbf{y}}+m Y_{l m}(\hat{\mathbf{r}}) \hat{\mathbf{z}}\right\},
\end{aligned}
$$

$$
\begin{aligned}
\mathbf{H}_{l m 2}(\mathbf{r})= & -\frac{i}{\sqrt{l(l+1)}} h_{l}(\beta r)\left\{\left[M_{l}^{m} Y_{l m+1}(\hat{\mathbf{r}})\right.\right. \\
& \left.+N_{l}^{m} Y_{l m-1}(\hat{\mathbf{r}})\right] \hat{\mathbf{x}}-i\left[M_{l}^{m} Y_{l m+1}(\hat{\mathbf{r}})\right. \\
& \left.\left.-N_{l}^{m} Y_{l m-1}(\hat{\mathbf{r}})\right] \hat{\mathbf{y}}+m Y_{l m}(\hat{\mathbf{r}}) \hat{\mathbf{z}}\right\}
\end{aligned}
$$

To prove Eqs. (25) and (26), we write

$$
\mathbf{u}_{\alpha}^{i n}(\mathbf{r})=\sum_{s \mathbf{g}} \mathbf{U}_{\alpha g}^{i n s} \exp \left(i \mathbf{k}_{\alpha g}^{s} \cdot \mathbf{r}\right)
$$

By using the identity

$$
\exp (i \mathbf{k} \cdot \mathbf{r})=\sum_{l m} 4 \pi i^{l}(-1)^{m} Y_{l-m}(\hat{\mathbf{k}}) j_{l}(k r) Y_{l m}(\hat{\mathbf{r}})
$$

we obtain 


$$
\begin{aligned}
\mathbf{U}_{\alpha g}^{i n s} & \exp \left(i \mathbf{k}_{\alpha g}^{s} \cdot \mathbf{r}\right) \\
& =\left(\mathbf{U}_{\alpha g}^{i n s} \cdot \frac{\mathbf{k}_{\alpha g}^{s}}{\alpha}\right) \frac{1}{i \alpha} \nabla\left[\exp \left(i \mathbf{k}_{\alpha g}^{s} \cdot \mathbf{r}\right)\right] \\
& =\left(\mathbf{U}_{\alpha g}^{i n s} \cdot \frac{\mathbf{k}_{\alpha g}^{s}}{\alpha}\right) \frac{1}{i \alpha} \nabla\left(\sum_{l m} 4 \pi(-1)^{m} Y_{l-m}\right. \\
& \left.\times\left(\hat{\mathbf{k}}_{\alpha g}^{s}\right) j_{l}(\alpha r) Y_{l m}(\hat{\mathbf{r}})\right) \\
& =\left(\mathbf{U}_{\alpha g}^{i n s} \cdot \mathbf{k}_{\alpha g}^{s}\right) \sum_{l m} \frac{4 \pi i^{l-1}(-1)^{m}}{\alpha} Y_{l-m}\left(\hat{\mathbf{k}}_{\alpha g}^{s}\right) \mathbf{J}_{l m 1}(\mathbf{r}),
\end{aligned}
$$

which gives

$$
\begin{aligned}
\mathbf{u}_{\alpha}^{i n}(\mathbf{r}) & =\sum_{s \mathbf{g}}\left(\mathbf{U}_{\alpha g}^{i n s} \cdot \mathbf{k}_{\alpha g}^{s}\right) \sum_{l m} \frac{4 \pi i^{l-1}(-1)^{m}}{\alpha} Y_{l-m}\left(\hat{\mathbf{k}}_{\alpha g}^{s}\right) \mathbf{J}_{l m 1}(\mathbf{r}) \\
& =\sum_{l m} \sum_{s \mathbf{g}}\left(\mathbf{U}_{\alpha g}^{i n s} \cdot \mathbf{k}_{\alpha g}^{s}\right) \frac{4 \pi i^{l-1}(-1)^{m}}{\alpha} Y_{l-m}\left(\hat{\mathbf{k}}_{\alpha g}^{s}\right) \mathbf{J}_{l m 1}(\mathbf{r}) .
\end{aligned}
$$

Comparing this equation with Eq. (24), we arrive at the first lines of Eqs. (25) and (26). To prove the second and third lines of Eqs. (25) and (26), we first write each term of the expansion

$$
\mathbf{u}_{\beta}^{i n}(\mathbf{r})=\sum_{s \mathbf{g}} \mathbf{U}_{\beta g}^{i n s} \exp \left(i \mathbf{k}_{\beta g}^{s} \cdot \mathbf{r}\right)
$$

as

$$
\mathbf{U}_{\beta g}^{i n s} \exp \left(i \mathbf{k}_{\beta g}^{s} \cdot \mathbf{r}\right)=\sum_{l m}\left[a_{l m 2}^{s g} \mathbf{J}_{l m 2}(\mathbf{r})+a_{l m 3}^{s g} \mathbf{J}_{l m 3}(\mathbf{r})\right] .
$$

By multiplying both sides of this equation by $\mathbf{J}_{l m 2}^{*}(\mathbf{r})$ and integrating on a spherical surface using the orthogonal relation

$$
\int \mathbf{J}_{l m 2}(\mathbf{r}) \cdot \mathbf{J}_{l^{\prime} m^{\prime} 2}^{*}(\mathbf{r}) d \hat{\mathbf{r}}=j_{l}^{2}(\beta r) \delta_{l l^{\prime}} \delta_{m m^{\prime}},
$$

we obtain

$$
j_{l}^{2}(\beta r) a_{l m 2}^{s g}=\int \mathbf{U}_{\beta g}^{i n s} \exp \left(i \mathbf{k}_{\beta g}^{s} \cdot \mathbf{r}\right) \cdot \mathbf{J}_{l m 2}^{*}(\mathbf{r}) d \hat{\mathbf{r}}
$$

From Eqs. (B1)-(B3), it follows that

$$
\begin{aligned}
a_{l m 2}^{s g}= & \mathbf{U}_{\beta g}^{i n s} \cdot \frac{4 \pi i^{l+1}(-1)^{m+1}}{\sqrt{l(l+1)}}\left\{\left[M_{l}^{m} Y_{l-(m+1)}\left(\hat{\mathbf{k}}_{\beta g}^{ \pm}\right)\right.\right. \\
& \left.+N_{l}^{m} Y_{l-(m-1)}\left(\hat{\mathbf{k}}_{\beta g}^{ \pm}\right)\right] \hat{\mathbf{x}}+i\left[M_{l}^{m} Y_{l-(m+1)}\left(\hat{\mathbf{k}}_{\beta g}^{ \pm}\right)\right. \\
& \left.\left.-N_{l}^{m} Y_{l-(m-1)}\left(\hat{\mathbf{k}}_{\beta g}^{ \pm}\right)\right] \hat{\mathbf{y}}-m Y_{l-m}\left(\hat{\mathbf{k}}_{\beta g}^{ \pm}\right) \hat{\mathbf{z}}\right\} .
\end{aligned}
$$

Since $\mathbf{J}_{l m 3}=(1 / \beta) \boldsymbol{\nabla} \times \mathbf{J}_{l m 2}$, we use $(1 / \beta) \boldsymbol{\nabla} \times$ to act on Eq. (B7) to obtain

$$
\frac{1}{\beta} \boldsymbol{\nabla} \times\left[\mathbf{U}_{\beta g}^{i n s} \exp \left(i \mathbf{k}_{\beta g}^{s} \cdot \mathbf{r}\right)\right]=\sum_{l m}\left[a_{l m 2}^{s g} \mathbf{J}_{l m 3}(\mathbf{r})+a_{l m 3}^{s g} \mathbf{J}_{l m 2}(\mathbf{r})\right] .
$$

Similarly, we have

$$
\begin{aligned}
j_{l}^{2}(\beta r) a_{l m 3}^{s g} & =\int \frac{1}{\beta} \nabla \times\left[\mathbf{U}_{\beta g}^{i n s} \exp \left(i \mathbf{k}_{\beta g}^{s} \cdot \mathbf{r}\right)\right] \cdot \mathbf{J}_{l m 2}^{*}(\mathbf{r}) d \hat{\mathbf{r}} \\
& =\int \frac{i}{\beta} \mathbf{k}_{\beta g}^{s} \times \mathbf{U}_{\beta g}^{i n s} \exp \left(i \mathbf{k}_{\beta g}^{s} \cdot \mathbf{r}\right) \cdot \mathbf{J}_{l m 2}^{*}(\mathbf{r}) d \hat{\mathbf{r}},
\end{aligned}
$$

which leads to

$$
\begin{aligned}
a_{l m 3}^{s g}= & \mathbf{U}_{\beta g}^{i n s} \cdot \frac{4 \pi i^{l}(-1)^{m+1}}{\sqrt{l(l+1)} \beta} \mathbf{k}_{\beta g}^{ \pm} \times\left\{\left[M_{l}^{m} Y_{l-(m+1)}\left(\hat{\mathbf{k}}_{\beta g}^{ \pm}\right)\right.\right. \\
& \left.+N_{l}^{m} Y_{l-(m-1)}\left(\hat{\mathbf{k}}_{\beta g}^{ \pm}\right)\right] \hat{\mathbf{x}}+i\left[M_{l}^{m} Y_{l-(m+1)}\left(\hat{\mathbf{k}}_{\beta g}^{ \pm}\right)\right. \\
& \left.\left.-N_{l}^{m} Y_{l-(m-1)}\left(\hat{\mathbf{k}}_{\beta g}^{ \pm}\right)\right] \hat{\mathbf{y}}-m Y_{l-m}\left(\hat{\mathbf{k}}_{\beta g}^{ \pm}\right) \hat{\mathbf{z}}\right\} .
\end{aligned}
$$

Substituting Eqs. (B10)-(B13) into Eq. (B7), and then substituting the resulting expression into Eq. (B6), we arrive at the second and third lines of Eqs. (25) and (26).

We now prove Eqs. (32) and (33). We first introduce the formula (see Pendry ${ }^{10}$ )

$$
\begin{aligned}
\sum_{\mathbf{R}} & \exp \left(i \mathbf{k}_{\|} \cdot \mathbf{R}\right) h_{l}(\kappa|\mathbf{r}-\mathbf{R}|) Y_{l m}(\hat{\mathbf{r}}-\hat{\mathbf{R}}) \\
= & \frac{2 \pi}{S \kappa} \sum_{\mathbf{g}} \frac{Y_{l m}(\hat{\mathbf{k}})}{\left(\kappa^{2}-\left|\mathbf{k}_{\|}+\mathbf{g}\right|^{2}\right)^{1 / 2}} \exp (i \mathbf{k} \cdot \mathbf{r}),
\end{aligned}
$$

where $\mathbf{k}=\left(\mathbf{k}_{\|}+\mathbf{g}, \sqrt{\kappa^{2}-\left|\mathbf{k}_{\|}+\mathbf{g}\right|^{2}}\right), \mathbf{R}$ is the two-dimensional lattice translation vector, and $\mathbf{g}$ is the reciprocal lattice vector. According to the definition of $\mathbf{H}_{l m 1}(\mathbf{r})$, we have

$$
\begin{aligned}
\sum_{\mathbf{R}} & \exp \left(i \mathbf{k}_{\|} \cdot \mathbf{R}\right) \mathbf{H}_{l m 1}(\mathbf{r}) \\
& =\sum_{\mathbf{R}} \exp \left(i \mathbf{k}_{\|} \cdot \mathbf{R}\right) \frac{1}{\alpha} \nabla\left[h_{l}(\kappa|\mathbf{r}-\mathbf{R}|) Y_{l m}(\hat{\mathbf{r}}-\hat{\mathbf{R}})\right] \\
& =\sum_{\mathbf{g}} \frac{2 \pi}{S} \frac{(-i)^{l-1}}{\alpha^{2}} \frac{Y_{l m}\left(\hat{\mathbf{k}}_{\alpha g}^{ \pm}\right)}{\left(\alpha^{2}-\left|\mathbf{k}_{\|}+\mathbf{g}\right|^{2}\right)^{1 / 2}} \mathbf{k}_{\alpha g}^{ \pm} \exp \left(i \mathbf{k}_{\alpha g}^{ \pm} \cdot \mathbf{r}\right) .
\end{aligned}
$$

Thus, we obtain the first lines of Eqs. (32) and (33). Similarly, by substituting the expression for $\mathbf{H}_{l m 2}(\mathbf{r})$, Eq. (B1), into $\sum_{\mathbf{R}} \exp \left(i \mathbf{k}_{\|} \cdot \mathbf{R}\right) \mathbf{H}_{l m 2}(\mathbf{r})$, we obtain the second lines in Eqs. (32) and (33). To obtain the last equations in Eqs. (32) and (33), we write

$$
\sum_{\mathbf{R}} \exp \left(i \mathbf{k}_{\|} \cdot \mathbf{R}\right) \mathbf{H}_{l m 3}(\mathbf{r})=\frac{1}{\beta} \boldsymbol{\nabla} \times\left(\sum_{\mathbf{R}} \exp \left(i \mathbf{k}_{\|} \cdot \mathbf{R}\right) \mathbf{H}_{l m 2}(\mathbf{r})\right)
$$

this leads directly to the desired expressions. 


\section{APPENDIX C}

In addition to the externally incident wave, the incident wave for the central scatterer includes the contributions from all the other scatterers in the scattering plane:

$$
\begin{aligned}
\sum_{i \neq 0} \sum_{l m \sigma} b_{l m \sigma}^{i} \mathbf{H}_{l m \sigma}^{i}\left(\mathbf{r}_{i}\right) & \\
= & \sum_{l m \sigma} b_{l m \sigma} \sum_{\mathbf{R}}^{\prime} \exp \left(i \mathbf{k}_{\|} \cdot \mathbf{R}\right) \mathbf{H}_{l m \sigma}(\mathbf{r}-\mathbf{R}) \\
= & \sum_{l m \sigma} b_{l m \sigma} \sum_{\mathbf{R}}^{\prime} \exp \left(i \mathbf{k}_{\|} \cdot \mathbf{R}\right) \sum_{l^{\prime} m^{\prime} \sigma^{\prime}} G_{l m \sigma l^{\prime} m^{\prime} \sigma^{\prime}} \\
& \times(-\mathbf{R}) \mathbf{J}_{l^{\prime} m^{\prime} \sigma^{\prime}}(\mathbf{r})=\sum_{l m \sigma} a_{l m \sigma^{\prime}}^{\prime} \mathbf{J}_{l m \sigma}(\mathbf{r}),
\end{aligned}
$$

where

$$
a_{l m \sigma}^{\prime}=\sum_{l^{\prime} m^{\prime} \sigma^{\prime}} b_{l^{\prime} m^{\prime} \sigma^{\prime}} G_{l^{\prime} m^{\prime} \sigma^{\prime} l m \sigma}\left(\mathbf{k}_{\|}\right)
$$

For the explicit expression of $G_{l m \sigma l^{\prime} m^{\prime} \sigma^{\prime}}\left(\mathbf{k}_{\|}\right)$, see Eq. (31). The total incident wave for the central scatterer in the plane is thus $\sum_{l m \sigma}\left(a_{l m \sigma}+a_{l m \sigma}^{\prime}\right) \mathbf{J}_{l m \sigma}(\mathbf{r})$. It follows that

$$
b_{l m \sigma}=\sum_{l^{\prime} m^{\prime} \sigma^{\prime}} t_{l m \sigma l^{\prime} m^{\prime} \sigma^{\prime}}\left(a_{l m \sigma}+a_{l m \sigma}^{\prime}\right),
$$

where $T=\left\{t_{l m \sigma l^{\prime} m^{\prime} \sigma^{\prime}}\right\}$ is the scattering matrix of the central scatterer. To write Eq. (C3) in the matrix form, we have

$$
B=T\left(A+A^{\prime}\right)=T\left[A+G^{T r}\left(\mathbf{k}_{\|}\right) B\right]
$$

thus

$$
B=Z A
$$

where the $Z$ matrix is defined as

$$
Z=\left[I-T G^{T r}\left(\mathbf{k}_{\|}\right)\right]^{-1} T
$$

with $I$ being the unit matrix.
*Current address: Crump Institute for Biological Imaging, Department of Molecular and Medical Pharmacology, UCLA School of Medicine, Los Angeles, CA 90095-1770.

${ }^{1}$ E. Yablonovitch, Phys. Rev. Lett. 58, 2059 (1987); E. Yablonovitch and T. J. Gmitter, ibid. 63, 1950 (1989).

${ }^{2}$ K. M. Ho, C. T. Chan, and C. M. Soukoulis, Phys. Rev. Lett. 65 , 3152 (1990); C. T. Chan, K. M. Ho, and C. M. Soukoulis, Europhys. Lett. 16, 563 (1991).

${ }^{3}$ X. D. Wang, X.-G. Zhang, Q. Yu, and B. N. Harmon, Phys. Rev. B 47, 4161 (1993).

${ }^{4}$ E. N. Economou and M. Sigalas, J. Acoust. Soc. Am. 95, 1734 (1994); A. D. Klironomos and E. N. Economou, Solid State Commun. 105, 327 (1998).

${ }^{5}$ M. S. Kushwaha, P. Halevi, and G. Martinez, Phys. Rev. B 49, 2313 (1994).

${ }^{6}$ M. Sigalas and E. N. Economou, Europhys. Lett. 36, 241 (1996).

${ }^{7}$ J. V. Sanchez-Perez, D. Caballero, R. Martinez-Sala, C. Rubio, J. Sanchez-Dehesa, F. Meseguer, J. Llinares, and F. Galvez, Phys. Rev. Lett. 80, 5325 (1998).

${ }^{8}$ F. R. Montero de Espinosa, E. Jimenez, and M. Torres, Phys. Rev. Lett. 80, 1208 (1998).

${ }^{9}$ See, for example, W. H. Butler, A. Gonis, and X. -G. Zhang, Phys. Rev. B 45, 11527 (1992); X. -G. Zhang and W. H. Butler, ibid. 46, 7433 (1992).

${ }^{10}$ J. B. Pendry, Low Energy Electron Diffraction (Academic, London, 1974); A. Modinos, Field, Thermionic, and Secondary Electron Emission Spectroscopy (Plenum, New York, 1984).

${ }^{11}$ A. Modinos, Physica A 141, 575 (1987); N. Stefanou, V. Karathanos, and A. Modinos, J. Phys.: Condens. Matter 4, 7389 (1992).

${ }^{12}$ P. C. Waterman, J. Acoust. Soc. Am. 60, 567 (1976); 63, 1320 (1978).

${ }^{13}$ A. Bostrom, J. Acoust. Soc. Am. 67, 1904 (1980); 67, 399 (1980) and the references therein.
${ }^{14}$ V. K. Varadan, V. V. Varadan, and Y. -H. Pao, J. Acoust. Soc. Am. 63, 1310 (1978).

${ }^{15}$ J. D. Achenbach and M. Kitahara, J. Acoust. Soc. Am. 80, 1209 (1986); 81, 595 (1987).

${ }^{16}$ S. L. Cheng, J.Appl. Mech. 36, 523 (1969); P. C. Waterman, J. Acoust. Soc. Am. 57, 791 (1975).

${ }^{17}$ P. M. Morse and H. Feshbach, Methods of Theoretical Physics (McGraw-Hill, New York, 1953), Chap. 13.

${ }^{18}$ The density of states is calculated with a linear tetrahedron method.

${ }^{19}$ W. M. Robertson, G. Arjavalingam, R. D. Meade, K. D. Brommer, A. M. Ragge, and J. D. Joannopoulos, Phys. Rev. Lett. 68, 2023 (1992).

${ }^{20}$ In the continuous-wave limit, multiple reflections in the support layer interfere to cause an additional modulation of the transmitted signal, which varies rapidly with frequency when the layer is thick, and appears as high-frequency noise, partially obscuring the fine structure in the transmission through the crystal alone. To eliminate this undesirable complication, our calculations were performed by introducing sufficient absorption in the support layer to reduce these multiple reflections to a negligible level. This was accomplished by making the longitudinal and shear moduli in the support layer complex, with the imaginary parts of the moduli equal to about $5 \%$ of the real parts. To determine the transmission through the crystal alone, the transmitted amplitude calculated under these conditions was normalized by the magnitude of the signal incident at the support-layer/ crystal interface; since this reference signal has traversed the attenuating support layer once, the normalization of the theoretical calculations is the same as that used for the experiments.

${ }^{21}$ M. Kafesaki and E. N. Economou, Phys. Rev. B 60, 11993 (1999).

${ }^{22}$ I. E. Psarobas, N. Stefanou, and A. Modinos, Phys. Rev. B (to be published). 